\title{
Possible Holocene excursion of the Earth's magnetic field in southern South America: New records from archaeological sites in Argentina
}

\author{
Hugo G. Nami ${ }^{1,2}$ \\ ${ }^{1}$ Laboratorio de Paleomagnetismo “Daniel A. Valencio”, Departamento de Ciencias Geológicas, Facultad de Ciencias Exactas, Físicas y Naturales, \\ Universidad de Buenos Aires, Ciudad Universitaria (Pabellón II), (1428) Buenos Aires, Argentina \\ ${ }^{2}$ Programa de Estudios Prehistóricos (CONICET), B. Mitre 1970 Piso 5 “A”, Buenos Aires (1039), República Argentina
}

(Received August 3, 1998; Revised November 18, 1998; Accepted December 17, 1998)

\begin{abstract}
Palaeomagnetic studies were carried out in several archaeological and palaeontological sites across Argentina and Chile. Remanence directions far removed from the present magnetic field, probably corresponding to a field excursion were recorded in several stratigraphic sections spaning $\sim 11$ to $2 \mathrm{ky} \mathrm{bp}$. Detailed palaeomagnetic data from 235 cores obtained in six sections from archaeological sites in Argentina are reported. Stability of the NRM was analyzed by progressive thermal and alternating field demagnetization and ChRM directions determined in most cases. Computed VGPs from those directions tend to be concentrated over North America, Europe, Eastern Asia and Africa. Using 275 VGPs a palaeomagnetic pole that is $\sim 20^{\circ}$ apart from the Earth's rotation's axis was obtained.
\end{abstract}

\section{Introduction}

Sedimentary deposits from archaeological sites, caves and rockshelters have been the subject of paleomagnetic research for many years. These investigations were useful in examining paleomagnetic secular variation (PSV) at different localities and periods (e.g. Elwood, 1971; Kopper and Creer, 1973; Creer and Kopper, 1974; Papmarinopoulous and Creer, 1983; Nöel and Thistlewood, 1989; Schmidt and Partridge, 1991). These studies also allowed the observation of geomagnetic field (GF) excursions and reversions (e.g. Kopper and Creer, 1976; Lovlie and Sandness, 1987; Parés and Pérez-González, 1995).

Recently, several palaeomagnetic results were obtained from cave sediments and archaeological sites from southern South America. They yielded evidence about PSV and a possible excursion of the GF occurred during the Holocene (Nami and Sinito, 1991, 1993; Nami, 1995; Nami et al., 1995, 1998; Sinito et al., 1997).

The study of the behaviour of the GF during excursions and reversals is extremely complex due to many variables affecting the palaeomagnetic recording process and the nature of the record (Merrill and McElhinny, 1983; Bogue and Merrill, 1992). However, though far from ideal, sedimentary sequences are suitable recording materials because they allow continuous stratigraphic analysis. Sediments have also a broad geographical distribution and are datable by ${ }^{14} \mathrm{C}$.

In this sense, the archaeological and paleontological sedimentary deposits located in Argentina and Chile have shown that they may contribute to the knowledge of the past GF behaviour (e.g. Nami, 1995; Nami et al., 1995; Sinito et al., 1997). Therefore, in this paper palaeomagnetic results obtained in six new sections from archaeological sites are re-

Copy right (C) The Society of Geomagnetism and Earth, Planetary and Space Sciences (SGEPSS); The Seismological Society of Japan; The Volcanological Society of Japan; The Geodetic Society of Japan; The Japanese Society for Planetary Sciences. ported. Additionally, a summary of the evidence of a probable GF excursion occurred in southern South America during the latest Pleistocene and Holocene is reported.

\section{The Sampling Sites and Age of Deposits}

For archaeological purposes, only sedimentary sequences corresponding to the end of the Pleistocene and to the Holocene $(\sim 11-0.5 \mathrm{ky} \mathrm{bp})$ were sampled. They are located in NW, NE and southern Argentina (Fig. 1). A brief description of the archaeological sites follow.

\subsection{Alero de las Circunferencias (Pintocayoc 1) (AC)}

AC $\left(22^{\circ} 56^{\prime} \mathrm{S}, 65^{\circ} 25^{\prime} \mathrm{W}\right)$ is a rockshelter located in the Humahuaca Department, Jujuy Province (NW Argentina). It was excavated by Hernández Llosas who identified a significant archaeological sequence in a $\sim 2.5 \mathrm{~m}$ deep deposit (Hernández Llosas et al., 1994). Seven layers were observed. These have different colours depending on the type of sedimentary matrix and vestige left: e.g. charcoal resulting from combustion, bones and other archaeological remains. The whole sequence is composed of fine sand (Bayarski et al., 1995).

Fifty-five samples were collected from the following layers: 5 (samples 1 to 15 ), 6 (16 to 54) and 7 (sample 55) (Fig. 2A).

Radiocarbon dates obtained from charcoal in the sampled section are as follows: layer 5: $7850 \pm 110$ (URU-0084), layer 6: $9190 \pm 110$ (LP-628), $9180 \pm 230$ (LP-449) and, layer 7: $10340 \pm 50$ (Beta-79849) and 10720 \pm 150 (LP-503) years b.p.

\subsection{Arroyo Yarará (AY)}

$\mathrm{AY}\left(26^{\circ} 0^{\prime} \mathrm{S}, 55^{\circ} 21^{\prime} \mathrm{W}\right)$ is an open air archaeological site located in the shorelines of the Yarará creek at Puerto Esperanza village, Misiones Province (NE Argentina).

During the spring of 1993 , a $0.6 \times 0.6 \mathrm{~m}$ test pit up to $0.6 \mathrm{~m}$ deep was excavated by the archaeologist J. Mujica and the author. Only one stratum of brown clay overlying 


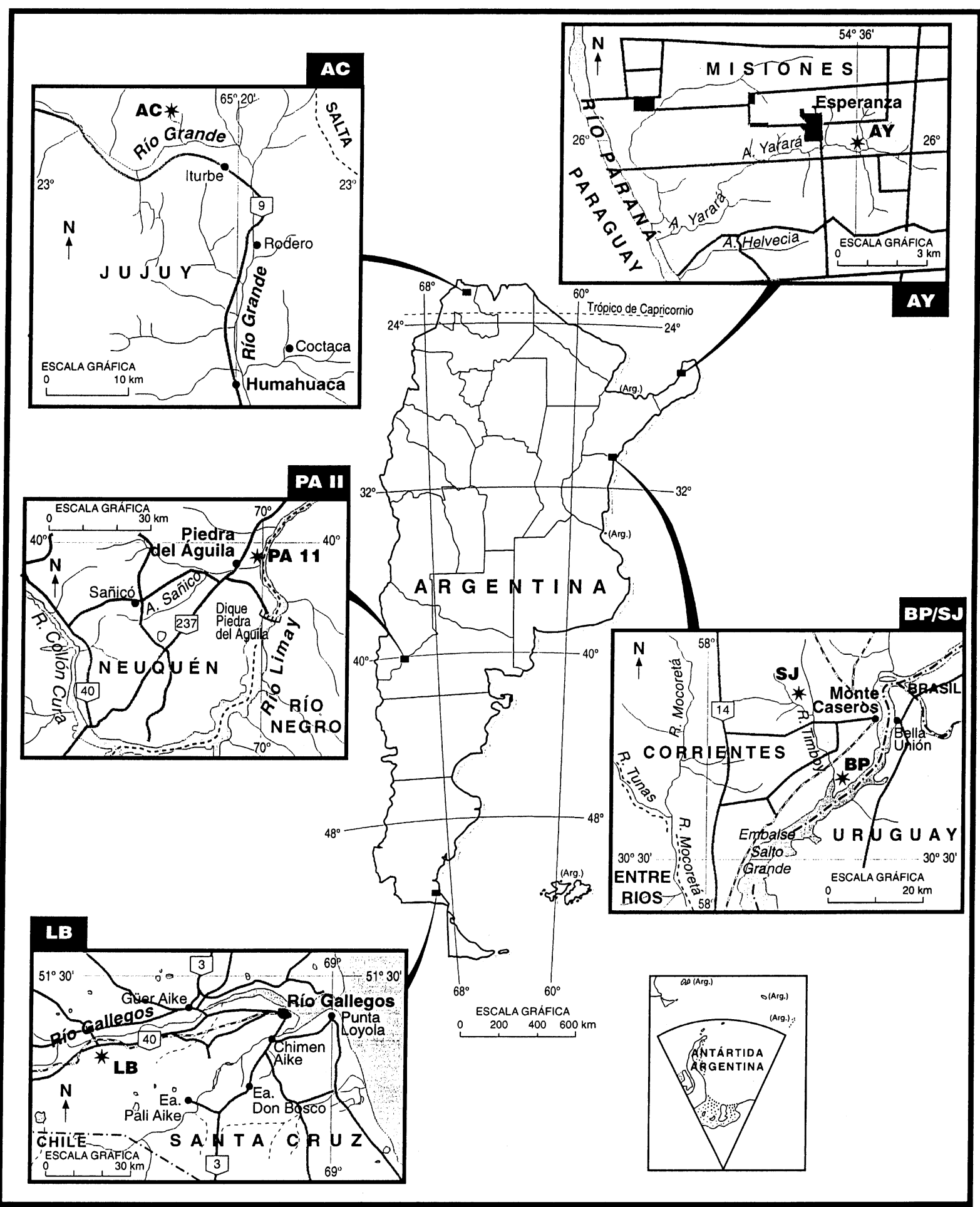

Fig. 1. Map of Argentina and location of the sampling sites mentioned in the text. AC: Alero de las Circunferencias, AY: Arroyo Yarará, BP: Barranca Pelada, SJ: San Juan, PA11: Piedra del Aguila 11 and, LB: Las Buitreras.

the basalt bedrock at this place was sampled $(n=14)$ for palaeomagnetic purposes (Fig. 2C).

Archaeological artifacts were found in the whole clay layer. Belonging from this archaeological level, there are surface archaeological artifacts, including lithic waste and stone tools commonly called "cleaves curves" or bifaces with boomerang form. According to Brazilian archaeolo- gists, these artifacts characterized the "Humaitá tradition" dated between $\sim 7$ and $1 \mathrm{ky} \mathrm{bp} \mathrm{(Schmitz,} \mathrm{1987).}$

\subsection{Barranca Pelada (BP)}

BP de Itacumbú $\left(30^{\circ} 15^{\prime} \mathrm{S}, 57^{\circ} 37^{\prime} \mathrm{W}\right)$ is located on the Uruguay river shoreline, $19 \mathrm{~km}$ to the South of Monte Caseros village, Corrientes Province (NE Argentina).

The sampled profile is $1 \mathrm{~m}$ thick and $1.10 \mathrm{~m}$ wide. Three 

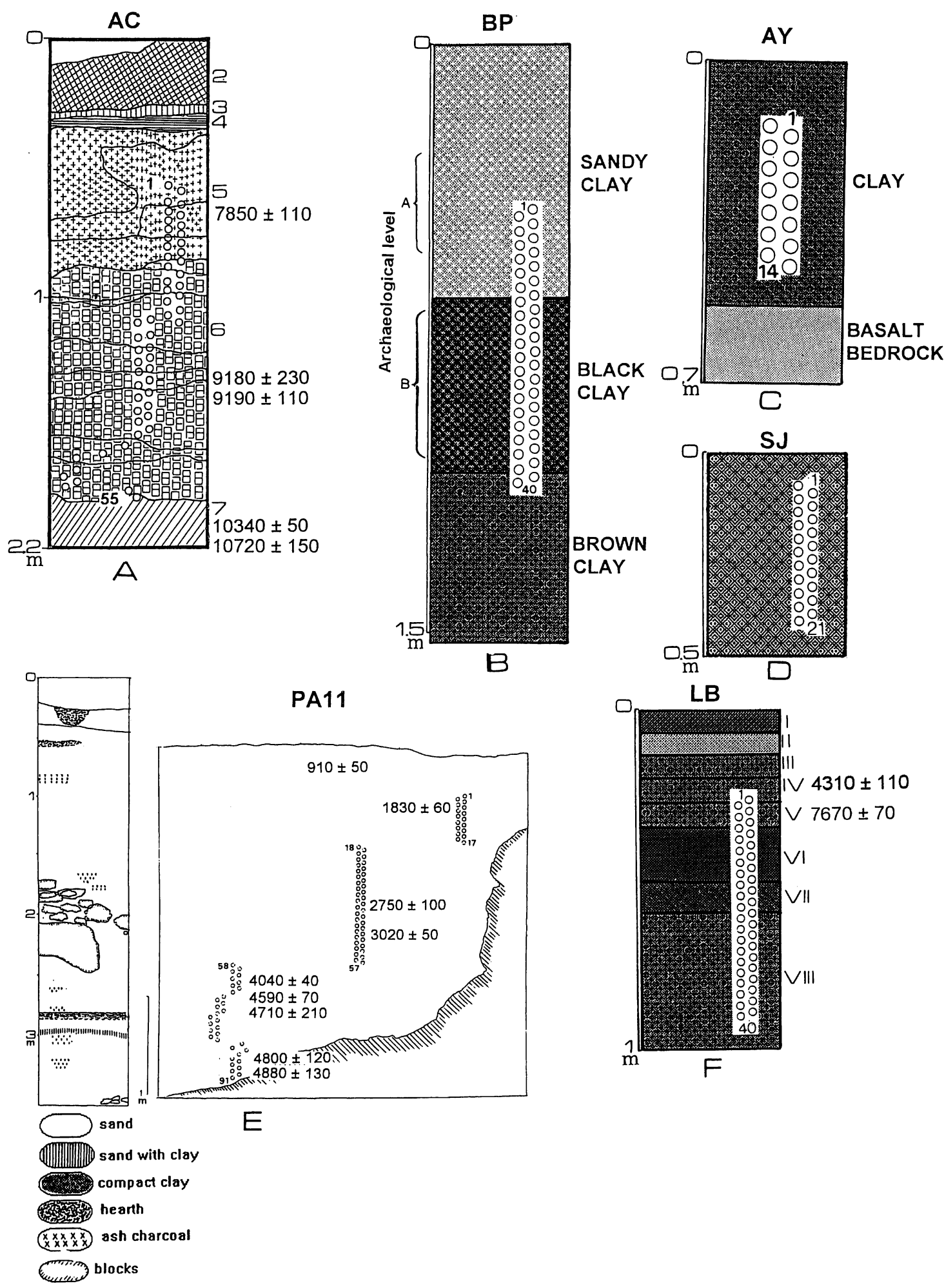

Fig. 2. Schematic stratigraphic profiles showing the sampled sections with location of the palaeomagnetic sampling related to stratigraphy. Only ${ }^{14} \mathrm{C}$ dates are given in the archaeological excavation. Numbers to the right of the sections show numbers of layers and those on the cylinders' top and bottom show the correlative order of the samples. A) Alero de las Circunferencias, B) Barranca Pelada, C) Arroyo Yarará, D) San Juan, E) Piedra del Aguila 11. Right: schematic stratigraphic profile. Left: Excavation section showing the sampling location, F) Las Buitreras.

natural strata can be seen I, II and III. Level I is mostly sand, and II and III are dark brown clays (Fig. 2B). The sediments have abundant fine grain quartz and some calcium carbonate with cubic structure (Smorczewski, 1994).
Forty samples were collected from the lower part of layer I (samples 1 to 11), layer II (12 to 37) and the upper portion of layer III (38 to 40).

According to Mujica (pers. comm., 1994) there are two 
archaeological levels. "A" in the upper section between $0.6-$ $0.8 \mathrm{~m}$ and "B" approximately at $0.9-1.05 \mathrm{~m}$ deep. Projectile points found in this site are similar to those from the "Umbú tradition" dated between 6 and 0.3 ky bp in many sites in SE Brazil, NE Argentina and Uruguay (Schmitz, 1987; Rodriguez, 1992).

\subsection{Estancia San Juan (SJ)}

$\mathrm{SJ}\left(30^{\circ} \mathrm{S}, 57^{\circ} 44^{\prime} \mathrm{W}\right)$ is located at a probable shoreline of an ancient bed of the Timboy creek, $22 \mathrm{~km}$ to the Northwest of Monte Caseros, Corrientes Province. The profile is $0.6 \mathrm{~m}$ thick and $1 \mathrm{~m}$ wide (Fig. 2D). Archaeological remains are found everywhere from the surface to $0.4 \mathrm{~m}$ deep.

The deposit is only one highly compacted dark gray stratum, probably a redeposited volcanic sediment. It is composed of medium to fine subangular to rounded quartz, chalcedony clasts and some angular lithics (Smorczewski, 1994). The samples $(n=21)$ were obtained from the surface to $0.4 \mathrm{~m}$ deep. Archaeological remains include bifacial artifacts and "bolas" stones. In the Salto Grande area, very close to Monte Caseros, similar remains belong to the middle Holocene (Rodriguez, 1992).

\subsection{Piedra del Aguila 11 (PA11)}

PA1 1 is a rockshelter about $9 \mathrm{~m}$ long and $5 \mathrm{~m}$ wide, located in the banks of the Limay River $\left(40^{\circ} 02^{\prime} \mathrm{S}, 70^{\circ} \mathrm{W}\right), 4 \mathrm{~km}$ to the north of the homonimous village in the Neuquén Province (SW Argentina). It was excavated by Sanguinetti de Bórmida and Curzio (1996) during several field seasons between 1985 and 1991. The excavations yielded an interesting archaeological sequence in a $\sim 3.60 \mathrm{~m}$ deep sedimentary deposit.

Samples $(n=91)$ were collected on the squares 24,4 and 12 of the southeastern profile that is $\sim 3.5 \mathrm{~m}$ deep and $\sim 4.5 \mathrm{~m}$ wide (Fig. 2E). From the surface to $3.6 \mathrm{~m}$ depth, the sediment is composed of gray sand with lenses of human hearths' ashes and silty sand or clay embedded in the matrix. The bottom of the excavation is composed of clear brown silty sand (Sanguinetti de Bórmida and Curzio, 1996).

The excavation was dated using ash charcoal collected at different levels. The radiometric dates are: $1830 \pm 60$

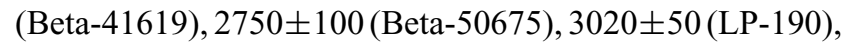

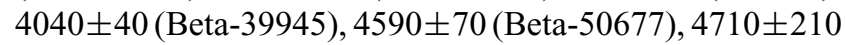
(Beta-50676), $4800 \pm 120$ (Beta-50678), $4880 \pm 130$ (Beta50679) (Sanguinetti de Bórmida and Curzio, 1996).

\subsection{Las Buitreras Cave (LB)}

$\mathrm{LB}\left(51^{\circ} 44.52^{\prime} \mathrm{W}, 70^{\circ} 10.28^{\prime} \mathrm{S}\right)$ is located $60 \mathrm{~km}$ to the west of the Río Gallegos city, Santa Cruz Province (southern Argentina). It is a big cave about $10 \mathrm{~m}$ long by $20 \mathrm{~m}$ wide with a $3 \mathrm{~m}$ deep sedimentary deposit. It was excavated by Sanguinetti de Bórmida in different field seasons during the '70 and '90s.

Eight layers were identified in the deposit (Sanguinetti de Bórmida, 1976). The sediments have a homogeneous mineralogical composition formed by basic volcanic and pyroclastic elements (Scasso, 1997). Layers I to V have the highest proportions of organic material suggesting abundant anthropogenic input (Eugenio, 1997).

Layers I and III were ${ }^{14} \mathrm{C}$ dated at $670 \pm 60$ (Beta-71985) and $750 \pm 60$ (Beta-71984) respectively, layer IV at $4310 \pm$ 110 (GAK-6051) and layer V was dated at $7670 \pm 70$ (CSIC372) years B.P. In layer VII there were blocks fallen down from the cave's wall, probably during a catastrophic move- ment. Similar phenomena occurred in other rockshelters in southern Patagonia. This event has been dated with a 9.5 ky bp minimum age (see Saxon, 1976; Nami, 1987, 1996; Prieto, 1991).

Extinct fauna bones of Mylodon cf. listai and Hippidion $s p$. were recovered from layers VI to VIII. Similar faunal remains from archaeological sites near this cave were dated as not younger than $10.5 \mathrm{ky} \mathrm{bp} \mathrm{(Nami} \mathrm{and} \mathrm{Nakamura,} \mathrm{1995;}$ Nami, 1996). This fact suggest that Ice Age animals in southern Patagonia became extinct $\sim 10 \mathrm{ky} \mathrm{bp}$ (Borrero, 1997).

Forty samples were collected in southern profile from layers IV (sample 1$), \mathrm{V}$ (2 to 7$)$, VI ( 8 to 15$)$, VII (16 to 20) and VIII (21 to 40) (Fig. 2F).

\section{Palaeomagnetic Analysis \\ 3.1 Sampling and laboratory procedures}

Samples were collected in cylindrical plastic and bronze containers $2.5 \mathrm{~cm}$ long by $2.5 \mathrm{~cm}$ in diameter in AC, AY and PA1 1 and $2 \mathrm{~cm}$ at BP, SJ and LB. The cylinders were carefully pushed into the sediment so that each specimen overlaps the previous one about $50 \%$ and their strike and dip were measured using a Brunton compass and inclinometer. Once removed, they were consolidated with sodium silicate. The stratigraphic position of each was carefully measured, registered, and sequentially numbered from the top to the bottom (Fig. 2). In some cases (AC, PA11 and LB) samples were not taken near the surface because the sediments were unconsolidated and they may have been disturbed by recent events, such as animal and/or human trampling.

Palaeomagnetic measurements were made using a $2 \mathrm{G}$ cryogenic magnetometer.

Stepwise alternating field (AF) and thermal demagnetization were used according to the nature of the sample containers and to determine the sediment response to different demagnetization procedures.

Sediments in plastic containers were subjected to progressive AF demagnetization in steps of 3, 6, 9, 12, 15, 20, 25, 30, 40 and $80 \mathrm{mT}$. Additional steps of 90,100 and 110 were used for some samples. Specimens contained in bronze cylinders were subjected to thermal demagnetization in a Schonstedt TSD- 1 demagnetizer in $50^{\circ} \mathrm{C}$ steps between $100^{\circ}$ and $500^{\circ} \mathrm{C}$, and additionally at $525^{\circ}$ and $550^{\circ} \mathrm{C}$.

The ChRM was isolated using principal component analysis (p.c.a.). The inherent scatter in directions was measured using the maximum angular deviation (MAD). This is the angle spanned by the standard deviation of the data perpendicular and parallel to the best fitting line (Kirschvink, 1980).

\subsection{Results}

According to the number of magnetic components and stability, remanence directions were qualified as: 1) "highly reliable" (HRe), with practically univectorial behaviour with decay towards the origin (e.g. Fig. 3a, 4f, 5b), 2) "moderately reliable" (MRe), with "noisy" behaviour during demagnetization and/or, multicomponent behaviour but a clear decay towards the origin or, well defined principal component but with erratic behaviour during final steps (e.g. Fig. 3d), 3) "poorly reliable" (PRe), with two or three components that could not be well isolated unless remagnetization circle analysis was used (Fig. 7), 4) "unreliable", with unstable behaviours. All samples rated as 3) and 4) were rejected 

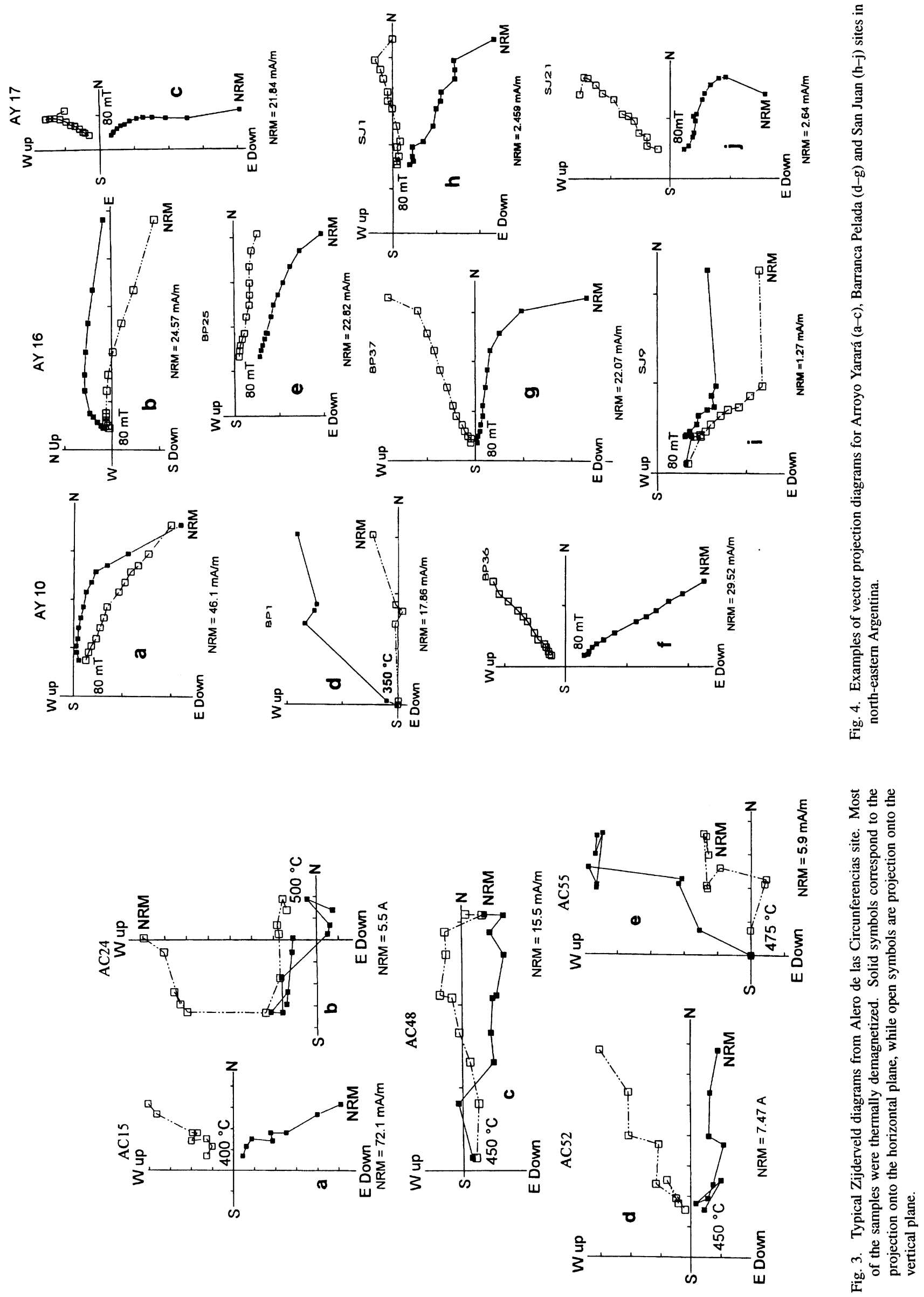

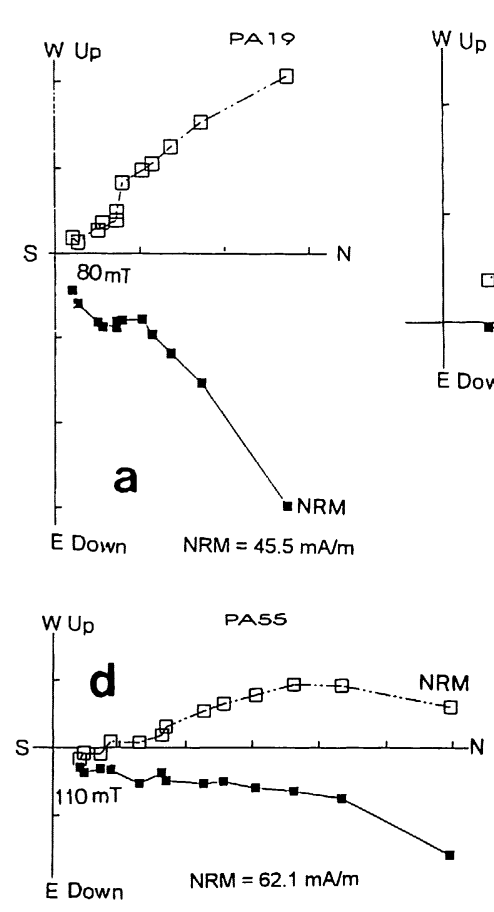
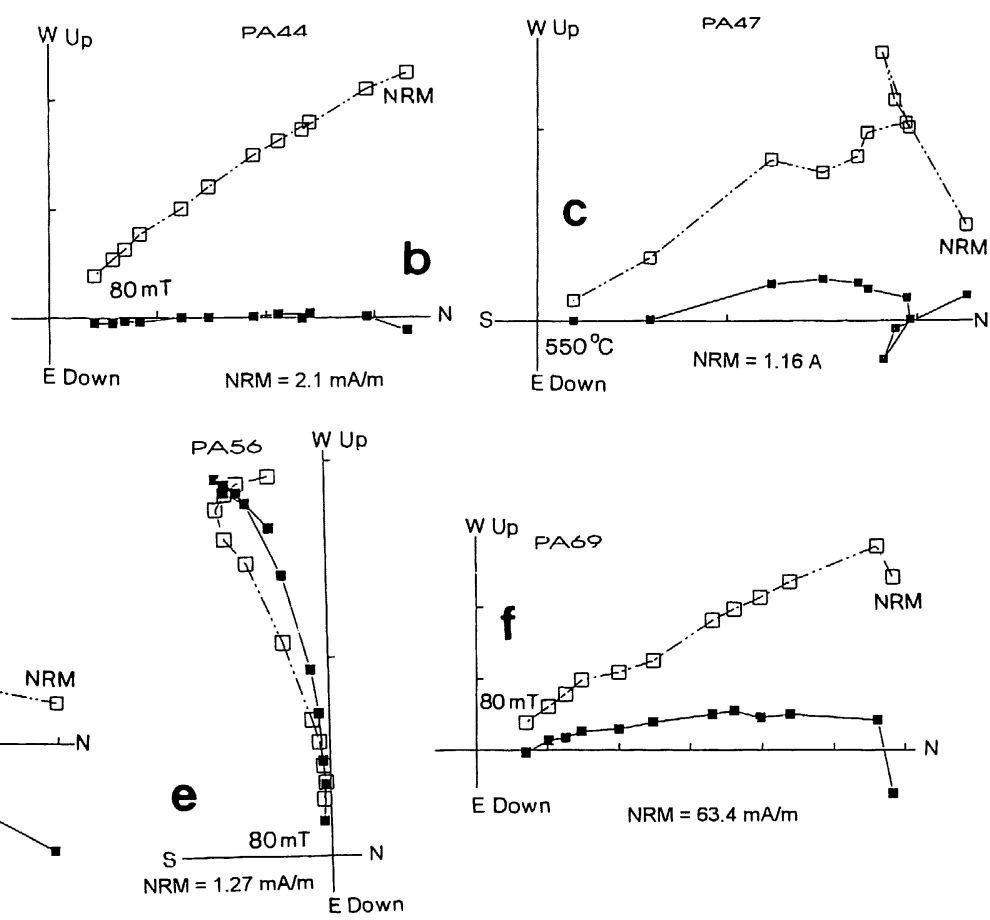

Fig. 5. Vector components diagrams showing the behaviour of typical samples cleaned using AF and thermal progressive demagnetization from Piedra del Aguila 11 site.
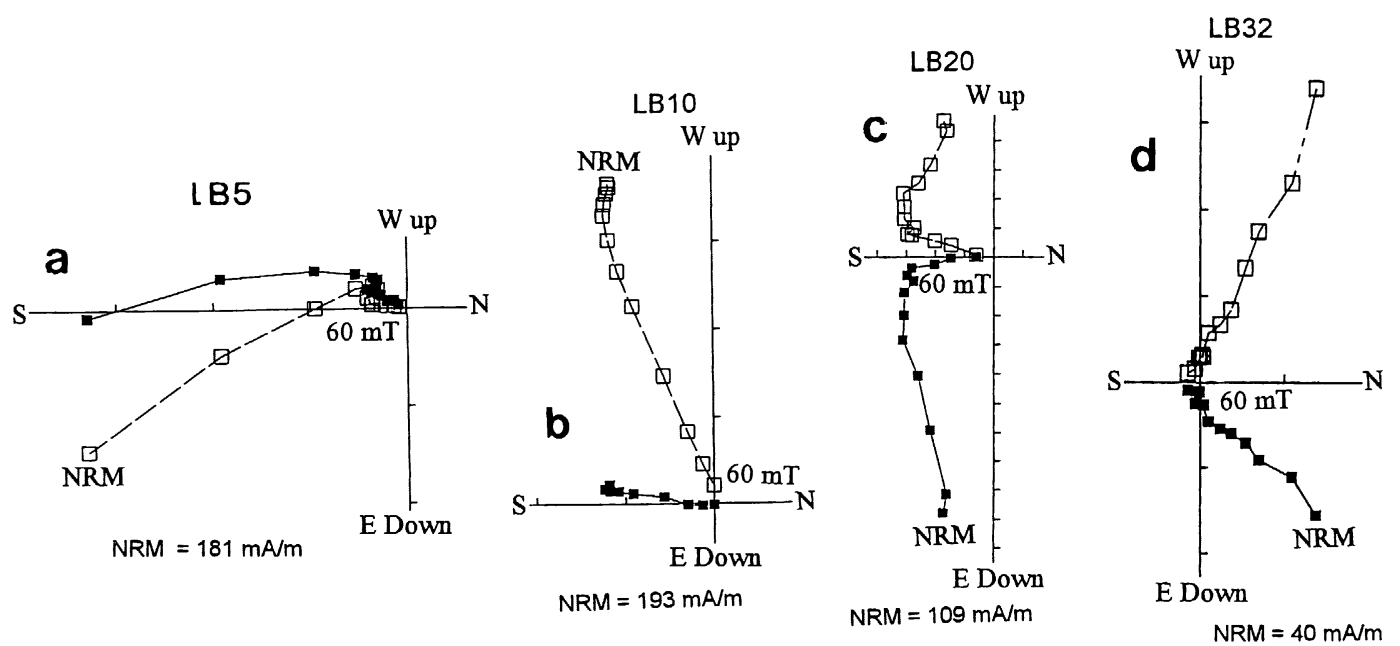

Fig. 6. Typical Zijderveld diagrams of stepwise demagnetization of four samples with normal, intermediate and reverse polarity from Las Buitreras site.

$(n=26)$.

In general samples from each site showed a common pattern with similar reliability. They are as follows:

AC: Samples from this site did not show significant differences under thermal and AF demagnetization procedures. However, due to the nature of the containers, most samples were processed using thermal cleaning.

Vector projection diagrams (VPD) (Zijderveld, 1967) illustrated in Fig. 3 show samples with either single component remanence with high inclination (AC15, Fig. 3a) and low inclination (sample AC48, Fig. 3c). Some samples had an overprint of the present geomagnetic field which was removed around $250^{\circ} \mathrm{C}$ (e.g. AC55, Fig. 3e) while others had two components, one with an anomalous southward direction (AC24, Fig. 3b).

Blocking temperatures between $450^{\circ}$ and $500^{\circ} \mathrm{C}$ (Fig. 3) suggest that the magnetic minerals belong to the titanomagnetite series (Tarling, 1983; Butler, 1992).

AY: Only AF demagnetization was used in this core, which proved to be highly efficient. Most samples had two components with the second one decaying to the origin in the VDP diagrams (Figs. 4a-c). Some samples showed low while oth- 


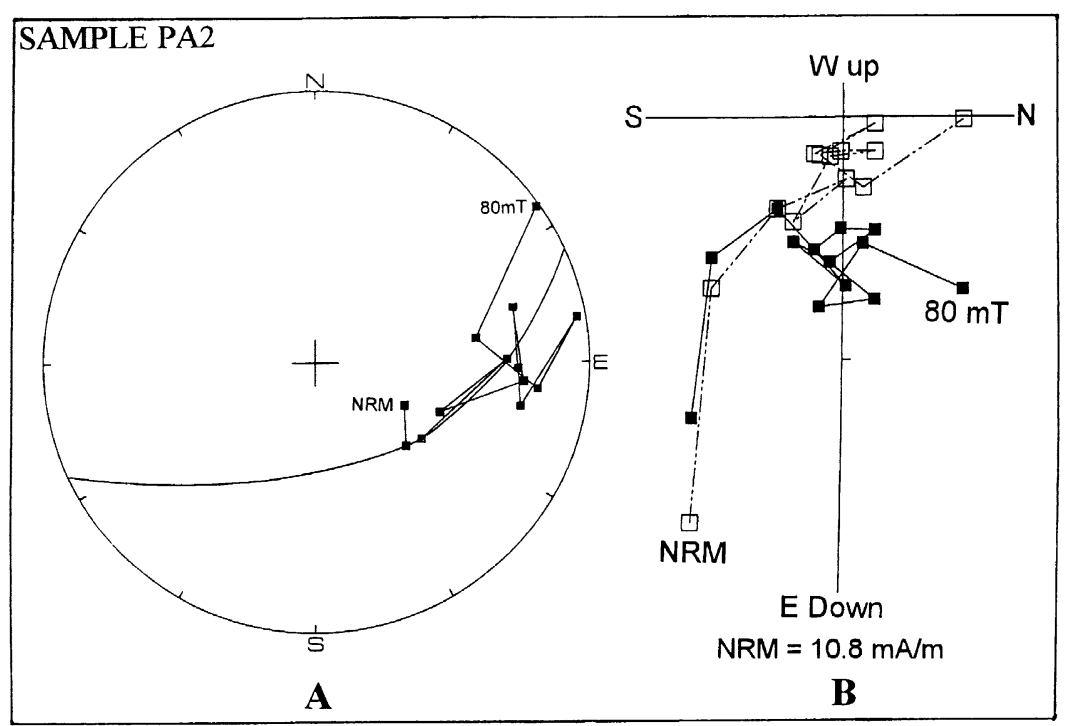

Fig. 7. Example of unreliable multicomponent sample to isolate directions from PA11 (\#2) moving in a maximum circle. A) Stereographic projection, B) Zijderveld diagram.

ers high inclination values, but in all cases negative (YA10 and YA17, Figs. 4a and 4c). A few ones, such as YA16, had an easterly declination and inclination (Fig. 4b).

BP: Here AF demagnetization was more efficient than thermal cleaning. Using this procedure some samples were very unstable and therefore, they were rejected. Like in the case of AY, Zijderveld diagrams show that samples had either low (BP25, Fig. 4e) or high inclinations with north-easterly (BP36, Fig. 4f) to south-easterly directions (BP36 and BP37, Figs. $4 \mathrm{f}-\mathrm{g}$ ).

Samples show an important drop in the NRM intensity at $350^{\circ} \mathrm{C}$ (BP1, Fig. 4d) suggesting the presence of some sulphides in their magnetic mineralogy, probably pyrrhotite (Tarling, 1983; Butler, 1992). This mineral can also be suggested because in those samples cleaned by AF, the NRM was removed at fields higher than $60 \mathrm{mT}$.

SJ: AF demagnetization was highly efficient in these samples. Except for a soft viscous component that was easily removed between 3 and $6 \mathrm{mT}$, the samples had univectorial behavior with decay towards the coordinate's origin. SJ1, SJ9 and SJ21 are examples of this kind of sample (Figs. 4h, $i$ and j).

PA11: Samples from this section did not show significant differences with AF and thermal demagnetization procedures. However, due to the nature of the containers, samples 40 to $42,46,47$ and 74 to 91 were processed using thermal cleaning. The remaining ones ( 1 to $39,44,45$ and 48 to 73 ) were demagnetized by AF cleaning.

Most of the samples were HRe with linear decay towards the origin (e.g. Figs. 5a-f), however some specimens showed a viscous component that was removed at $100-200^{\circ} \mathrm{C}$ (e.g. PA47, Fig. 5c).

Many samples showed either high (PA19, Fig. 5a) or low negative inclinations (PA55, Fig. 5d). A few showed a southwesterly direction (PA56, Fig. 5e).

Blocking temperatures at $450^{\circ}$ to $550^{\circ} \mathrm{C}$ (Fig. $5 \mathrm{c}$ ) suggest that the magnetic carrier belong to the titanomagnetite series
Table 1. Ranges of MAD values discriminated by sites.

\begin{tabular}{crccc}
\hline Site & $\begin{array}{r}0-5^{\circ} \\
(\%)\end{array}$ & $\begin{array}{c}5.1-10^{\circ} \\
(\%)\end{array}$ & $\begin{array}{c}10.1-15^{\circ} \\
(\%)\end{array}$ & $\begin{array}{c}15.1-20^{\circ} \\
(\%)\end{array}$ \\
\hline AC & 2.8 & 33.3 & 63.9 & - \\
AY & 71.5 & 28.5 & - & - \\
BP & 38.2 & 41.2 & 17.7 & 2.9 \\
SJ & 4.8 & 85.7 & 9.5 & - \\
PA11 & 36.7 & 48.9 & 10 & 4.4 \\
LB & 22.5 & 55 & 2.5 & 2.5 \\
\hline
\end{tabular}

(Tarling, 1983; Butler, 1992).

LB: Samples subjected to thermal demagnetization were highly unstable. However, AF cleaning showed to be the more efficient for this core. As illustrated in Fig. 6, VPD shows clear southerly directions for samples LB5, LB10 and LB20 (Figs. 6a-c) while others show northerly directions and negative inclinations (e.g. LB32, Fig. 6d).

As represented in Table 1, MAD values were very low. The number and intervals of demagnetization steps used to isolate the ChRM and VGPs positions are described in Table 2. All the sections show normal and intermediate polarity directions far from the present GF, while reversed polarities were recorded at $\mathrm{AC}$ and $\mathrm{LB}$.

Figures 8 to 12 summarize the changes in the declinations and inclinations of the ChRM isolated for the sections reported here. The more conspicuous long declination and inclination departures are depicted between dashed lines and indicated with arrows.

AC magnetogram (Fig. 8) show strong swings in declination between samples 20 and 50 dated between $\sim 10$ and $\sim 7 \mathrm{ky} \mathrm{bp}$. There is also an important correlative shift toward higher negative values in inclination (over $40^{\circ}$ ). 
Table 2. Values of characteristic remanent magnetization (ChRM), virtual geomagnetic pole positions and intervals of selected ChRM for each sample. Negatives values show negative inclination or VGP located in the southern hemisphere. Intervals of selected ChRM are given in $\mathrm{mT}$ or ${ }^{\circ} \mathrm{C}$ according thermal $(\mathrm{T})$ or alternating field (AF) demagnetization procedures used. Or. $=$ Origin in the Zijderveld diagram.

\begin{tabular}{|c|c|c|c|c|c|c|}
\hline Sample & $D^{\circ}$ & $I^{\circ}$ & Long. E & Lat. & Int. ChRM & Cleaning \\
\hline 3 & 34 & -68 & 80 & 52 & $0-200$ & $\mathrm{~T}$ \\
\hline 6 & 33 & -40 & 31 & 60 & 450-Or. & $\mathrm{T}$ \\
\hline 7 & 17 & -38 & 22 & 74 & $0-$ Or. & $\mathrm{T}$ \\
\hline 8 & 51 & -27 & 24 & 41 & $250-450$ & $\mathrm{~T}$ \\
\hline 9 & 55 & -37 & 33 & 39 & $100-250$ & $\mathrm{~T}$ \\
\hline 10 & 5 & 16 & 304 & 59 & 400-Or. & $\mathrm{T}$ \\
\hline 11 & 37 & -35 & 26 & 56 & 250-Or. & $\mathrm{T}$ \\
\hline 12 & 63 & -30 & 30 & 31 & $350-450$ & $\mathrm{~T}$ \\
\hline 13 & 59 & -21 & 23 & 33 & 350-Or. & $\mathrm{T}$ \\
\hline 14 & 348 & -78 & 121 & 45 & 200-Or. & $\mathrm{T}$ \\
\hline 15 & 61 & -33 & 32 & 33 & 0-Or. & $\mathrm{T}$ \\
\hline 17 & 14 & -19 & 343 & 71 & 100-Or. & $\mathrm{T}$ \\
\hline 18 & 21 & 6 & 336 & 57 & $250-500$ & $\mathrm{~T}$ \\
\hline 20 & 24 & -24 & 4 & 65 & $100-200$ & $\mathrm{~T}$ \\
\hline 24 & 213 & -6 & 170 & -47 & $300-450$ & $\mathrm{~T}$ \\
\hline 25 & 25 & 5 & 342 & 55 & 350-Or. & $\mathrm{T}$ \\
\hline 26 & 8 & -53 & 83 & 77 & $300-400$ & $\mathrm{~T}$ \\
\hline 27 & 345 & -15 & 198 & 47 & $0-250$ & $\mathrm{~T}$ \\
\hline 28 & 262 & -47 & 176 & 4 & $300-425$ & $\mathrm{~T}$ \\
\hline 29 & 3 & -21 & 308 & 78 & 350-Or. & $\mathrm{T}$ \\
\hline 30 & 16 & -11 & 352 & 65 & 300-Or. & $\mathrm{T}$ \\
\hline 32 & 334 & -6 & 239 & 58 & $0-250$ & $\mathrm{~T}$ \\
\hline 33 & 289 & -50 & 180 & 27 & $0-300$ & $\mathrm{~T}$ \\
\hline 35 & 275 & -5 & 204 & 6 & 400-Or. & $\mathrm{T}$ \\
\hline 36 & 350 & 13 & 275 & 59 & 500-Or. & $\mathrm{T}$ \\
\hline 38 & 45 & -30 & 24 & 47 & $12-25$ & $\mathrm{AF}$ \\
\hline 39 & 358 & 19 & 291 & 57 & $9-25$ & $\mathrm{AF}$ \\
\hline 41 & 46 & -39 & 34 & 48 & 0-Or. & $\mathrm{T}$ \\
\hline 44 & 25 & -24 & 6 & 63 & $0-200$ & $\mathrm{~T}$ \\
\hline 45 & 11 & -30 & 353 & 78 & 0-Or. & $\mathrm{T}$ \\
\hline 48 & 11 & -8 & 326 & 68 & 200-Or. & $\mathrm{T}$ \\
\hline 50 & 359 & -24 & 289 & 80 & $0-400$ & $\mathrm{~T}$ \\
\hline 51 & 39 & 13 & 352 & 42 & $350-$ Or. & $\mathrm{T}$ \\
\hline 52 & 6 & -25 & 326 & 79 & 0-Or. & $\mathrm{T}$ \\
\hline 54 & 37 & -25 & 16 & 54 & $250-400$ & $\mathrm{~T}$ \\
\hline 55 & 312 & 7 & 228 & 36 & 300-Or. & $\mathrm{T}$ \\
\hline
\end{tabular}

\begin{tabular}{|c|c|c|c|c|c|c|}
\hline Sample & $D^{\circ}$ & $I^{\circ}$ & Long. E & Lat. & Int. ChRM & Cleaning \\
\hline 1 & 357 & -1 & 298 & 64 & $25-60$ & $\mathrm{AF}$ \\
\hline 2 & 359 & -17 & 301 & 73 & $25-$ Or. & $\mathrm{AF}$ \\
\hline 3 & 342 & -42 & 217 & 74 & $25-$ Or. & $\mathrm{AF}$ \\
\hline 4 & 43 & 5 & 302 & 39 & 30-Or. & $\mathrm{AF}$ \\
\hline 5 & 9 & -19 & 334 & 72 & $25-$ Or. & $\mathrm{AF}$ \\
\hline 6 & 353 & -18 & 282 & 72 & 15-Or. & $\mathrm{AF}$ \\
\hline 7 & 3 & -20 & 316 & 74 & $40-60$ & $\mathrm{AF}$ \\
\hline 8 & 43 & -2 & 11 & 42 & 50-Or. & $\mathrm{AF}$ \\
\hline 9 & 5 & 25 & 312 & 50 & $20-60$ & $\mathrm{AF}$ \\
\hline 10 & 3 & -31 & 322 & 80 & $9-40$ & $\mathrm{AF}$ \\
\hline 11 & 332 & 25 & 266 & 42 & $60-$ Or. & $\mathrm{AF}$ \\
\hline 12 & 60 & -66 & 77 & 39 & 15-Or. & $\mathrm{AF}$ \\
\hline 13 & 42 & -21 & 22 & 48 & 40-Or. & $\mathrm{AF}$ \\
\hline 14 & 46 & -47 & 49 & 49 & $30-$ Or. & $\mathrm{AF}$ \\
\hline
\end{tabular}

Table 2. (continued).

\begin{tabular}{|c|c|c|c|c|c|c|}
\hline Sample & $D^{\circ}$ & $I^{\circ}$ & Long. E & Lat. & Int. ChRM & Cleaning \\
\hline 1 & 318 & -0.3 & 241 & 40 & 150-Or. & $\mathrm{T}$ \\
\hline 2 & 37 & -24 & 13 & 51 & $0-250$ & $\mathrm{~T}$ \\
\hline 8 & 284 & 25 & 230 & 5 & 350-Or. & $\mathrm{T}$ \\
\hline 9 & 10 & -30 & 338 & 73 & $0-200$ & $\mathrm{~T}$ \\
\hline 10 & 346 & 7 & 279 & 54 & $250-400$ & $\mathrm{~T}$ \\
\hline 11 & 54 & -19 & 22 & 36 & $0-150$ & $\mathrm{~T}$ \\
\hline 13 & 350 & -23 & 273 & 70 & 250-Or. & $\mathrm{T}$ \\
\hline 14 & 6 & -17 & 317 & 68 & 100-Or. & $\mathrm{T}$ \\
\hline 15 & 25 & -20 & 357 & 59 & $100-250$ & $\mathrm{~T}$ \\
\hline 16 & 7 & -17 & 321 & 67 & 200-Or. & $\mathrm{T}$ \\
\hline 17 & 31 & -25 & 8 & 57 & 30-Or. & $\mathrm{AF}$ \\
\hline 18 & 350 & -50 & 223 & 81 & $200-300$ & $\mathrm{~T}$ \\
\hline 19 & 355 & -23 & 288 & 71 & 30-Or. & $\mathrm{AF}$ \\
\hline 20 & 338 & -43 & 223 & 70 & 30-Or. & $\mathrm{AF}$ \\
\hline 21 & 57 & -23 & 26 & 35 & $0-150$ & $\mathrm{~T}$ \\
\hline 22 & 15 & -16 & 338 & 63 & 0-Or. & $\mathrm{AF}$ \\
\hline 23 & 16 & -19 & 342 & 65 & 3-Or. & $\mathrm{AF}$ \\
\hline 24 & 7 & -12 & 318 & 65 & 3-Or. & $\mathrm{AF}$ \\
\hline 25 & 20 & 6 & 336 & 51 & 3-Or. & $\mathrm{AF}$ \\
\hline 26 & 9 & -9 & 322 & 63 & $250-400$ & $\mathrm{~T}$ \\
\hline 27 & 17 & 17 & 327 & 48 & 3-Or. & $\mathrm{AF}$ \\
\hline 28 & 11 & -12 & 328 & 63 & 6-Or. & $\mathrm{AF}$ \\
\hline 29 & 24 & -23 & 357 & 61 & 6-Or. & $\mathrm{AF}$ \\
\hline 30 & 347 & 4 & 279 & 55 & $150-300$ & $\mathrm{~T}$ \\
\hline 31 & 71 & -30 & 37 & 24 & $300-400$ & $\mathrm{~T}$ \\
\hline 32 & 29 & 12 & 344 & 45 & 0-Or. & $\mathrm{AF}$ \\
\hline 33 & 16 & -35 & 358 & 72 & 3-Or. & $\mathrm{AF}$ \\
\hline 34 & 38 & -16 & 8 & 48 & 3-Or. & $\mathrm{AF}$ \\
\hline 35 & 6 & -60 & 99 & 78 & $3-100$ & $\mathrm{AF}$ \\
\hline 36 & 59 & -23 & 27 & 33 & 0-Or. & $\mathrm{AF}$ \\
\hline 37 & 10 & -22 & 330 & 69 & 6-Or. & $\mathrm{AF}$ \\
\hline 38 & 17 & -42 & 16 & 74 & $0-200$ & $\mathrm{~T}$ \\
\hline 39 & 28 & -47 & 329 & 26 & 3-Or. & $\mathrm{AF}$ \\
\hline 40 & 342 & -66 & 153 & 67 & $3-30$ & $\mathrm{AF}$ \\
\hline
\end{tabular}

\begin{tabular}{|c|c|c|c|c|c|c|}
\hline Sample & $D^{\circ}$ & $I^{\circ}$ & Long. E & Lat. & Int. ChRM & Cleaning \\
\hline 1 & 19 & 3 & 335 & 54 & 25-Or. & $\mathrm{AF}$ \\
\hline 2 & 360 & 1 & 302 & 59 & $3-15$ & $\mathrm{AF}$ \\
\hline 3 & 338 & -38 & 230 & 68 & 3-Or. & $\mathrm{AF}$ \\
\hline 4 & 48 & -52 & 47 & 49 & 6-Or. & $\mathrm{AF}$ \\
\hline 5 & 8 & -33 & 335 & 76 & 6-Or. & $\mathrm{AF}$ \\
\hline 6 & 8 & -22 & 326 & 70 & $15-40$ & $\mathrm{AF}$ \\
\hline 7 & 14 & -20 & 339 & 66 & 3-Or. & $\mathrm{AF}$ \\
\hline 8 & 11 & -49 & 34 & 80 & 3-Or. & $\mathrm{AF}$ \\
\hline 9 & 32 & -46 & 333 & 25 & 3-Or. & $\mathrm{AF}$ \\
\hline 10 & 11 & 24 & 318 & 46 & $6-30$ & $\mathrm{AF}$ \\
\hline 11 & 1 & -30 & 306 & 76 & 6-Or. & $\mathrm{AF}$ \\
\hline 12 & 341 & -28 & 249 & 67 & 3-Or. & $\mathrm{AF}$ \\
\hline 13 & 25 & -42 & 22 & 68 & 3-Or. & $\mathrm{AF}$ \\
\hline 14 & 17 & -50 & 39 & 75 & $3-60$ & $\mathrm{AF}$ \\
\hline 15 & 16 & -22 & 344 & 66 & $3-80$ & $\mathrm{AF}$ \\
\hline 16 & 1 & -19 & 305 & 70 & 3-Or. & $\mathrm{AF}$ \\
\hline 17 & 14 & 20 & 323 & 47 & 3-Or. & $\mathrm{AF}$ \\
\hline 18 & 33 & -46 & 35 & 61 & 3-Or. & $\mathrm{AF}$ \\
\hline 19 & 353 & -32 & 274 & 76 & $6-50$ & $\mathrm{AF}$ \\
\hline 20 & 45 & -18 & 15 & 43 & $3-20$ & $\mathrm{AF}$ \\
\hline 21 & 25 & -39 & 18 & 66 & 3-Or. & $\mathrm{AF}$ \\
\hline
\end{tabular}


Table 2. (continued).

PA11

\begin{tabular}{|c|c|c|c|c|c|c|}
\hline Sample & $D^{\circ}$ & $I^{\circ}$ & Long. E & Lat. & Int. ChRM & Cleaning \\
\hline 1 & 41 & -15 & 350 & 41 & $12-80$ & $\mathrm{AF}$ \\
\hline 3 & 64 & -17 & 9 & 25 & $0-25$ & $\mathrm{AF}$ \\
\hline 4 & 49 & -12 & 355 & 34 & 15-Or. & $\mathrm{AF}$ \\
\hline 5 & 53 & -15 & 356 & 32 & $15-30$ & $\mathrm{AF}$ \\
\hline 6 & 24 & -23 & 333 & 55 & $3-15$ & $\mathrm{AF}$ \\
\hline 7 & 20 & 27 & 330 & 59 & $15-25$ & $\mathrm{AF}$ \\
\hline 8 & 233 & -84 & 122 & 32 & $0-15$ & $\mathrm{AF}$ \\
\hline 9 & 49 & -5 & 353 & 32 & 50-Or. & $\mathrm{AF}$ \\
\hline 10 & 78 & -8 & 15 & 12 & $0-12$ & $\mathrm{AF}$ \\
\hline 11 & 72 & 1 & 8 & 14 & $0-12$ & $\mathrm{AF}$ \\
\hline 12 & 78 & -15 & 18 & 14 & $3-20$ & $\mathrm{AF}$ \\
\hline 13 & 90 & 0 & 20 & 0 & 60-Or. & $\mathrm{AF}$ \\
\hline 14 & 40 & -25 & 353 & 46 & $30-60$ & $\mathrm{AF}$ \\
\hline 15 & 37 & -34 & 358 & 52 & 3-Or. & $\mathrm{AF}$ \\
\hline 16 & 54 & -11 & 0 & 30 & 20-Or. & $\mathrm{AF}$ \\
\hline 17 & 60 & -42 & 22 & 38 & 60-Or. & $\mathrm{AF}$ \\
\hline 18 & 53 & -19 & 3 & 34 & $0-40$ & $\mathrm{AF}$ \\
\hline 19 & 57 & -17 & 5 & 31 & 30-Or. & $\mathrm{AF}$ \\
\hline 20 & 245 & -71 & 143 & 20 & $60-$ Or. & $\mathrm{AF}$ \\
\hline 21 & 75 & -4 & 12 & 13 & $0-20$ & $\mathrm{AF}$ \\
\hline 22 & 66 & 9 & 8 & 21 & $0-15$ & $\mathrm{AF}$ \\
\hline 23 & 41 & -33 & 0 & 49 & $3-60$ & $\mathrm{AF}$ \\
\hline 24 & 38 & -21 & 350 & 45 & 30-Or. & $\mathrm{AF}$ \\
\hline 25 & 17 & -33 & 342 & 62 & 20-Or. & $\mathrm{AF}$ \\
\hline 26 & 9 & -29 & 309 & 64 & 3-Or. & $\mathrm{AF}$ \\
\hline 27 & 341 & -29 & 309 & 64 & 3-Or. & $\mathrm{AF}$ \\
\hline 28 & 338 & -45 & 230 & 67 & 3-Or. & $\mathrm{AF}$ \\
\hline 29 & 12 & -47 & 333 & 74 & 3-Or. & $\mathrm{AF}$ \\
\hline 30 & 352 & -44 & 261 & 74 & 3-Or. & $\mathrm{AF}$ \\
\hline 31 & 29 & -37 & 350 & 59 & 3-Or. & $\mathrm{AF}$ \\
\hline 32 & 4 & -30 & 300 & 66 & 3-Or. & $\mathrm{AF}$ \\
\hline 33 & 8 & -41 & 315 & 72 & 3-Or. & $\mathrm{AF}$ \\
\hline 34 & 128 & -41 & 338 & 68 & 3-Or. & $\mathrm{AF}$ \\
\hline 35 & 359 & -43 & 286 & 75 & 3-Or. & $\mathrm{AF}$ \\
\hline 36 & 2 & -43 & 297 & 75 & 3-Or. & $\mathrm{AF}$ \\
\hline 37 & 1 & -44 & 292 & 75 & 3-Or. & $\mathrm{AF}$ \\
\hline 38 & 4 & -30 & 298 & 66 & 3-Or. & $\mathrm{AF}$ \\
\hline 39 & 355 & -32 & 277 & 67 & 3-Or. & $\mathrm{AF}$ \\
\hline 40 & 16 & -20 & 290 & 50 & 300-Or. & $\mathrm{T}$ \\
\hline 41 & 358 & -40 & 284 & 73 & 450-Or. & $\mathrm{T}$ \\
\hline 42 & 8 & -8 & 304 & 53 & $350-500$ & $\mathrm{~T}$ \\
\hline 43 & 8 & -41 & 313 & 72 & 3-Or. & $\mathrm{AF}$ \\
\hline 44 & 359 & -35 & 287 & 69 & 3-Or. & $\mathrm{AF}$ \\
\hline 45 & 2 & -27 & 295 & 64 & 3-Or. & $\mathrm{AF}$ \\
\hline 46 & 336 & -12 & 255 & 45 & $250-450$ & $\mathrm{~T}$ \\
\hline 47 & 352 & 28 & 273 & 64 & 300-Or. & $\mathrm{T}$ \\
\hline 48 & 352 & -37 & 268 & 69 & $12-80$ & $\mathrm{AF}$ \\
\hline 49 & 6 & -30 & 307 & 65 & 3-Or. & $\mathrm{AF}$ \\
\hline 50 & 30 & -16 & 339 & 48 & 40-Or. & $\mathrm{AF}$ \\
\hline 51 & 1 & -16 & 291 & 58 & 40-Or. & $\mathrm{AF}$ \\
\hline 52 & 2 & -14 & 294 & 57 & 9-Or. & $\mathrm{AF}$ \\
\hline 53 & 35 & -30 & 353 & 51 & 30-Or. & $\mathrm{AF}$ \\
\hline 54 & 3 & -32 & 298 & 67 & 25-Or. & $\mathrm{AF}$ \\
\hline 55 & 42 & 8 & 342 & 32 & 9-Or. & $\mathrm{AF}$ \\
\hline 56 & 268 & -28 & 187 & 8 & $40-80$ & $\mathrm{AF}$ \\
\hline 57 & 16 & -35 & 324 & 61 & 30-Or. & $\mathrm{AF}$ \\
\hline 58 & 345 & -25 & 261 & 60 & 12-Or. & $\mathrm{AF}$ \\
\hline
\end{tabular}

Table 2. (continued).

\begin{tabular}{|c|c|c|c|c|c|c|}
\hline Sample & $D^{\circ}$ & $I^{\circ}$ & Long. E & Lat. & Int. ChRM & Cleaning \\
\hline 59 & 358 & -30 & 286 & 66 & 3-Or. & $\mathrm{AF}$ \\
\hline 60 & 349 & -21 & 270 & 59 & 3-Or. & $\mathrm{AF}$ \\
\hline 61 & 346 & 3 & 269 & 47 & 6-Or. & $\mathrm{AF}$ \\
\hline 62 & 36 & -24 & 349 & 48 & 3-Or. & $\mathrm{AF}$ \\
\hline 63 & 3 & -42 & 300 & 74 & 3-Or. & $\mathrm{AF}$ \\
\hline 64 & 32 & -35 & 352 & 55 & 12-Or. & $\mathrm{AF}$ \\
\hline 65 & 355 & -46 & 269 & 76 & 3-Or. & $\mathrm{AF}$ \\
\hline 66 & 351 & -20 & 272 & 59 & 3-Or. & $\mathrm{AF}$ \\
\hline 67 & 60 & -24 & 10 & 31 & 3-Or. & $\mathrm{AF}$ \\
\hline 68 & 356 & -33 & 279 & 68 & 3-Or. & $\mathrm{AF}$ \\
\hline 69 & 354 & -27 & 278 & 64 & 3-Or. & $\mathrm{AF}$ \\
\hline 70 & 6 & -30 & 303 & 66 & 3-Or. & $\mathrm{AF}$ \\
\hline 71 & 2 & -21 & 294 & 61 & 6-Or. & $\mathrm{AF}$ \\
\hline 72 & 12 & -37 & 320 & 68 & 3-Or. & $\mathrm{AF}$ \\
\hline 73 & 1 & -33 & 292 & 68 & 3-Or. & $\mathrm{AF}$ \\
\hline 74 & 4 & -33 & 299 & 68 & 100-Or. & $\mathrm{T}$ \\
\hline 75 & 13 & -38 & 335 & 31 & 300-Or. & $\mathrm{T}$ \\
\hline 76 & 272 & -45 & 180 & 18 & $400-500$ & $\mathrm{~T}$ \\
\hline 77 & 94 & -55 & 50 & 20 & $200-300$ & $\mathrm{~T}$ \\
\hline 78 & 344 & 9 & 269 & 43 & $350-450$ & $\mathrm{~T}$ \\
\hline 79 & 360 & -34 & 290 & 68 & 250-Or. & $\mathrm{T}$ \\
\hline 80 & 16 & -27 & 317 & 56 & 300-Or. & $\mathrm{T}$ \\
\hline 81 & 341 & -37 & 244 & 65 & 400-Or. & $\mathrm{T}$ \\
\hline 82 & 341 & -34 & 248 & 63 & 200-Or. & $\mathrm{T}$ \\
\hline 83 & 6 & -19 & 301 & 56 & 0-300 & $\mathrm{T}$ \\
\hline 84 & 254 & -38 & 173 & 2 & $100-250$ & $\mathrm{~T}$ \\
\hline 85 & 28 & -14 & 335 & 49 & $450-500$ & $\mathrm{~T}$ \\
\hline 86 & 38 & -50 & 15 & 58 & 150-Or. & $\mathrm{T}$ \\
\hline 87 & 356 & -14 & 283 & 57 & $250-350$ & $\mathrm{~T}$ \\
\hline 88 & 358 & -31 & 285 & 67 & 300-Or. & $\mathrm{T}$ \\
\hline 89 & 340 & -41 & 237 & 66 & $300-550$ & $\mathrm{~T}$ \\
\hline 90 & 345 & -47 & 234 & 74 & 150-Or. & $\mathrm{T}$ \\
\hline 91 & 360 & -41 & 289 & 74 & $150-300$ & $\mathrm{~T}$ \\
\hline
\end{tabular}

\begin{tabular}{|c|c|c|c|c|c|c|}
\hline Sample & $D^{\circ}$ & $I^{\circ}$ & Long. E & Lat. & Int. ChRM & Cleaning \\
\hline 1 & 61 & 9 & 354 & 14 & $3-30$ & $\mathrm{AF}$ \\
\hline 2 & 350 & -52 & 264 & 70 & $6-30$ & $\mathrm{AF}$ \\
\hline 3 & 35 & -57 & 4 & 62 & 30-Or. & $\mathrm{AF}$ \\
\hline 4 & 90 & -57 & 46 & 29 & 6-Or. & $\mathrm{AF}$ \\
\hline 5 & 175 & 27 & 102 & -53 & $0-40$ & $\mathrm{AF}$ \\
\hline 6 & 29 & -29 & 334 & 48 & $6-30$ & $\mathrm{AF}$ \\
\hline 7 & 347 & -40 & 266 & 60 & $3-40$ & $\mathrm{AF}$ \\
\hline 8 & 355 & 31 & 285 & 22 & 40-Or. & $\mathrm{AF}$ \\
\hline 9 & 292 & -68 & 173 & 49 & 50-Or. & $\mathrm{AF}$ \\
\hline 10 & 279 & -70 & 163 & 44 & 3-Or. & $\mathrm{AF}$ \\
\hline 11 & 41 & -77 & 69 & 65 & $3-80$ & $\mathrm{AF}$ \\
\hline 12 & 3 & -52 & 298 & 71 & $3-80$ & $\mathrm{AF}$ \\
\hline 13 & 48 & -24 & 353 & 35 & $12-30$ & $\mathrm{AF}$ \\
\hline 14 & 66 & -22 & 8 & 24 & 0-Or. & $\mathrm{AF}$ \\
\hline 15 & 78 & -44 & 28 & 27 & $20-40$ & $\mathrm{AF}$ \\
\hline 16 & 13 & -48 & 318 & 66 & 3-Or. & $\mathrm{AF}$ \\
\hline 17 & 42 & -50 & 3 & 53 & $0-$ Or. & $\mathrm{AF}$ \\
\hline 18 & 99 & -43 & 42 & 14 & $12-30$ & $\mathrm{AF}$ \\
\hline 19 & 23 & -79 & 86 & 70 & $4-80$ & $\mathrm{AF}$ \\
\hline 20 & 169 & -16 & 97 & -30 & 30-Or. & $\mathrm{AF}$ \\
\hline 21 & 60 & -64 & 36 & 51 & 3-Or. & $\mathrm{AF}$ \\
\hline 22 & 352 & -73 & 147 & 83 & $3-50$ & $\mathrm{AF}$ \\
\hline
\end{tabular}


Table 2. (continued).

\begin{tabular}{crccccc}
\hline Sample & \multicolumn{1}{c}{$D^{\circ}$} & $I^{\circ}$ & Long. E & Lat. & Int. ChRM & Cleaning \\
\hline 23 & 360 & -74 & 110 & 81 & $3-60$ & $\mathrm{AF}$ \\
24 & 18 & -76 & 79 & 75 & $3-60$ & $\mathrm{AF}$ \\
25 & 4 & -64 & 316 & 84 & $9-25$ & $\mathrm{AF}$ \\
26 & 10 & -70 & 46 & 83 & $3-40$ & $\mathrm{AF}$ \\
27 & 27 & -65 & 13 & 71 & $3-12$ & $\mathrm{AF}$ \\
28 & 28 & -51 & 347 & 62 & $3-12$ & $\mathrm{AF}$ \\
29 & 31 & -67 & 27 & 70 & $3-20$ & $\mathrm{AF}$ \\
30 & 353 & -16 & 280 & 46 & $12-25$ & $\mathrm{AF}$ \\
31 & 50 & -57 & 18 & 53 & $0-20$ & $\mathrm{AF}$ \\
32 & 43 & -55 & 9 & 56 & $3-0 r$. & $\mathrm{AF}$ \\
33 & 66 & -55 & 27 & 41 & $3-80$ & $\mathrm{AF}$ \\
34 & 31 & -63 & 13 & 68 & $3-O r$. & $\mathrm{AF}$ \\
35 & 350 & -67 & 211 & 83 & $0-0 r$. & $\mathrm{AF}$ \\
36 & 306 & -40 & 214 & 40 & $6-20$ & $\mathrm{AF}$ \\
37 & 51 & -62 & 27 & 55 & $0-60$ & $\mathrm{AF}$ \\
38 & 7 & -53 & 307 & 71 & $3-30$ & $\mathrm{AF}$ \\
39 & 349 & -54 & 262 & 71 & $3-50$ & $\mathrm{AF}$ \\
40 & 15 & -53 & 327 & 69 & $3-20$ & $\mathrm{AF}$ \\
\hline
\end{tabular}

BP shows gradual changes toward positive inclinations between samples 1 and 11 and particularly between 20 and 35 (Fig. 9). AY shows transitional negative to positive inclinations between samples 3 and 12 (Fig. 10B). SJ core also recorded a transitional zone defined by a gradual, negative to positive change in inclination between samples 8 and 14 (Fig. 10A). Relative chronologies for AY, BP and SJ suggest that the observed GF anomalous behaviour occurred in the middle to late Holocene.

In PA11, a significant but gentle eastward shift in the declination (over $40^{\circ}$ ) and a less conspicuous shallowing of the inclination can be observed in the upper part of the section between $\sim 2.5$ and 1.9 ky bp (Fig. 11). Figure 12 also illustrates the long pulses in declination and inclination during a possible excursion of the GF. Like in Mylodon Cave (Nami, 1995), LB records suggest a shift from normal to intermediate and reversed positions during the Late Pleistocene to early Holocene (Fig. 12). This fact occurs between samples 1 and 21 in layer IV to VII, that is between $\sim 4$ and $\sim 10 \mathrm{ky} \mathrm{bp}$.

Figures 13 and 14 illustrates the direction of the ChRM and corresponding virtual geomagnetic pole (VGP) positions for each section. It can be seen that generally VGP's are located

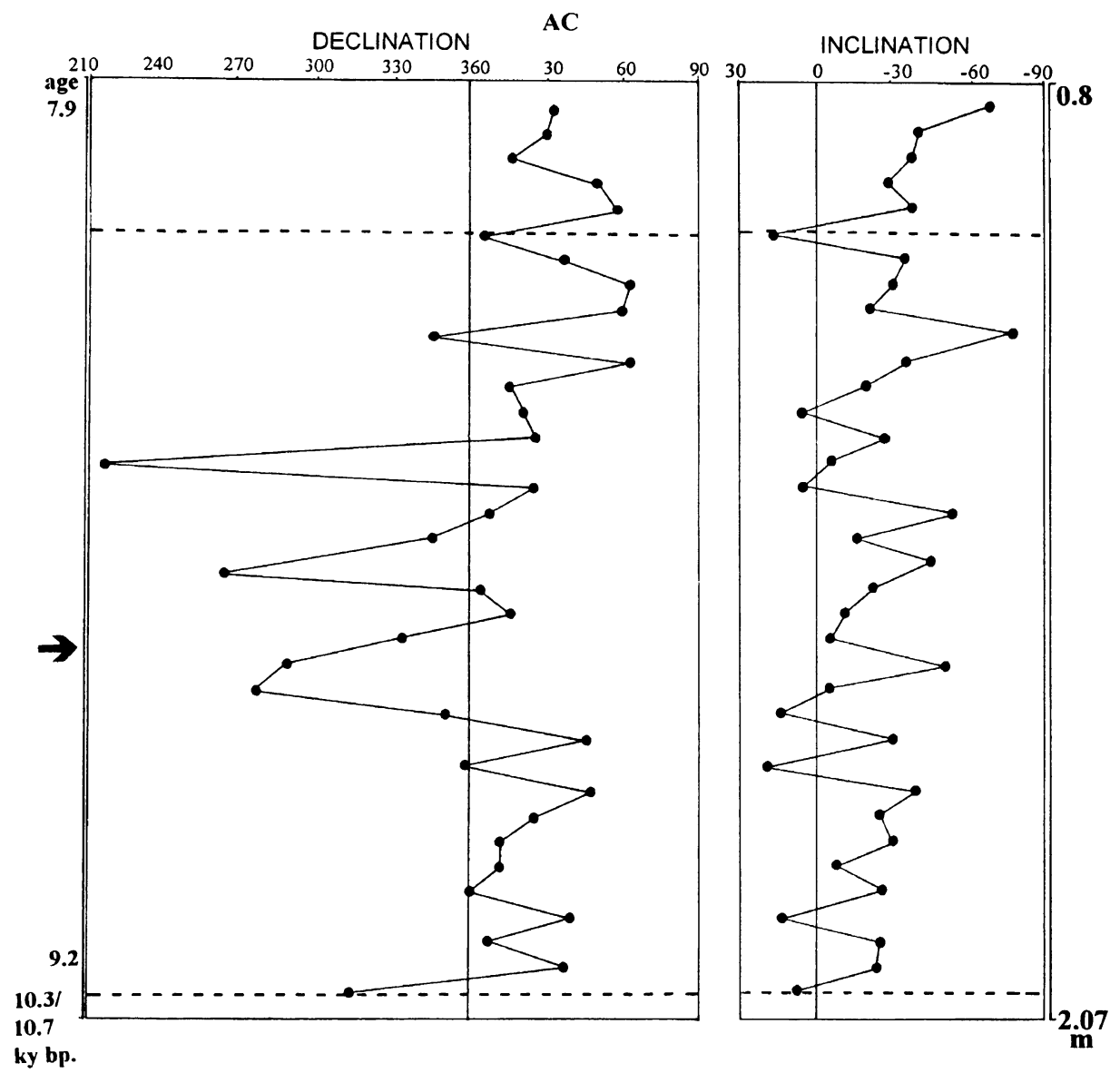

Fig. 8. Stratigraphic presentation of the profile of declination and inclination from Alero de las Circunferencias. The more conspicuous long declination and inclination departures are indicated by arrows between dashed lines. Chronologies in the sections are only shown when they have direct absolute dates by ${ }^{14} \mathrm{C}$. Samples' depths are indicated to the right of the diagrams (Figs. 8 to 12). 


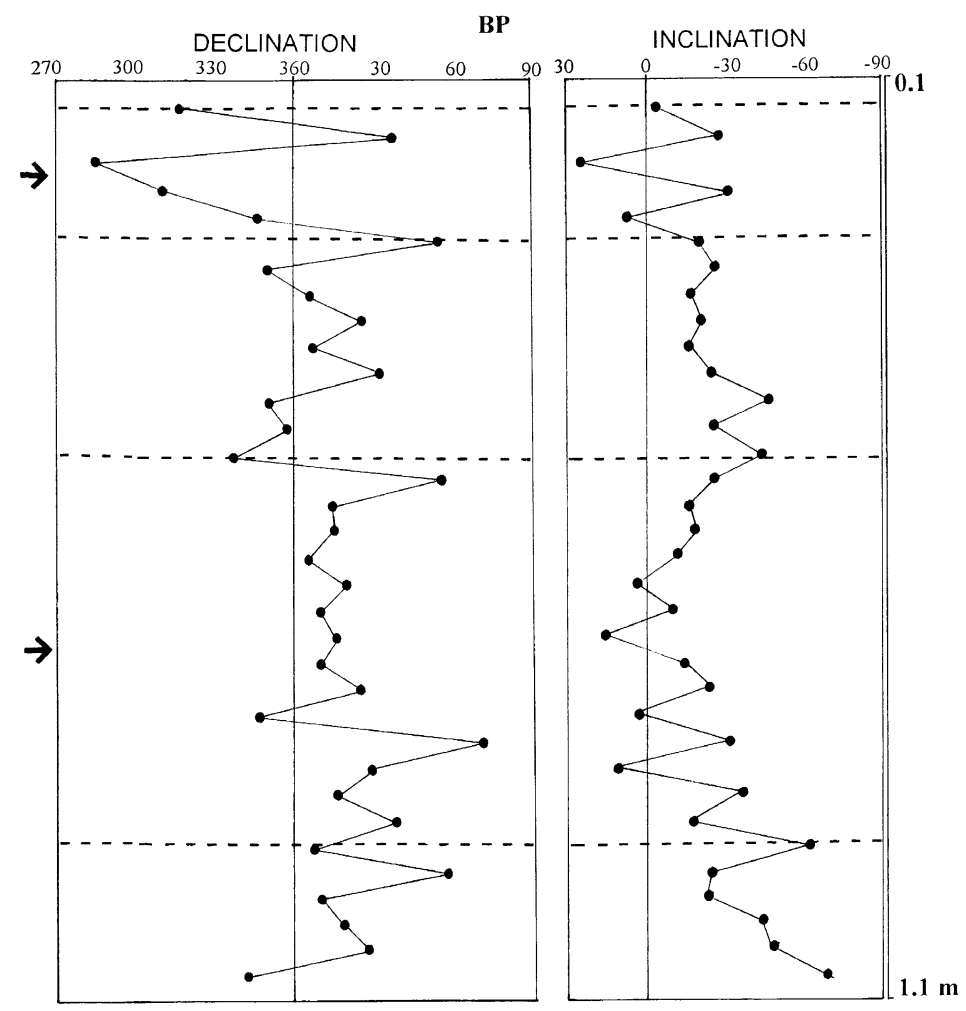

Fig. 9. Declination and inclination logs for the sequence of Barranca Pelada archaeological site.

in North America, Europe, Eastern Asia and Africa (Fig. 15).

Finally, Fig. 17B shows one geomagnetic pole computed from all VGP's located within a $40^{\circ}$ window around the mean geomagnetic pole (cf. McElhinny et al., 1974). It was isolated with 275 VGP obtained from samples at the Don Ariel Cave (Nami, 1994), the Mylodon Cave (Nami, 1995) and all the sites reported in this paper (Figs. 16, 17A). The mean geomagnetic pole is located at $336.7^{\circ} \mathrm{W}$. Long., $68.65^{\circ} \mathrm{S}$. Lat. with $\left(\mathrm{A}_{95}\right) 8.2^{\circ}$. This palaeopole shows a $\sim 20^{\circ}$ difference with respect to the Earth's rotation's axis (Fig. 17B). This fact, might suggest that PSV was not averaged, even considering that the data encompass $\sim 8$ kyr time span (cf. Hyodo et al., 1993, p. 692). If this difference is a geomagnetic problem, these results might be partially attributed to the far-sided effect (Merrill and McElhinny, 1983).

\section{Summary of Holocene Anomalous Magnetic Direction Records in Southern South America and Conclusion}

The results reported in this paper show that many samples of Late-Pleistocene and Holocene deposits from Argentina recorded directions far removed from the present magnetic field, probably corresponding to a field excursion that occurred at sometime between 11 and $\leq 2 \mathrm{ky}$ bp. This fact agrees with previous results obtained in other archaeological and palaeontological sites in Argentina and Chile, where remanence directions corresponding to an obliquely normal, obliquely reversed and reversed field polarity were found (Nami, 1994, 1995; Nami et al., 1995, 1998; Sinito et al., 1997). This situation suggests that the "Mylodon excursion" had a regional extent. Both shifts with N-R and R-N direc-

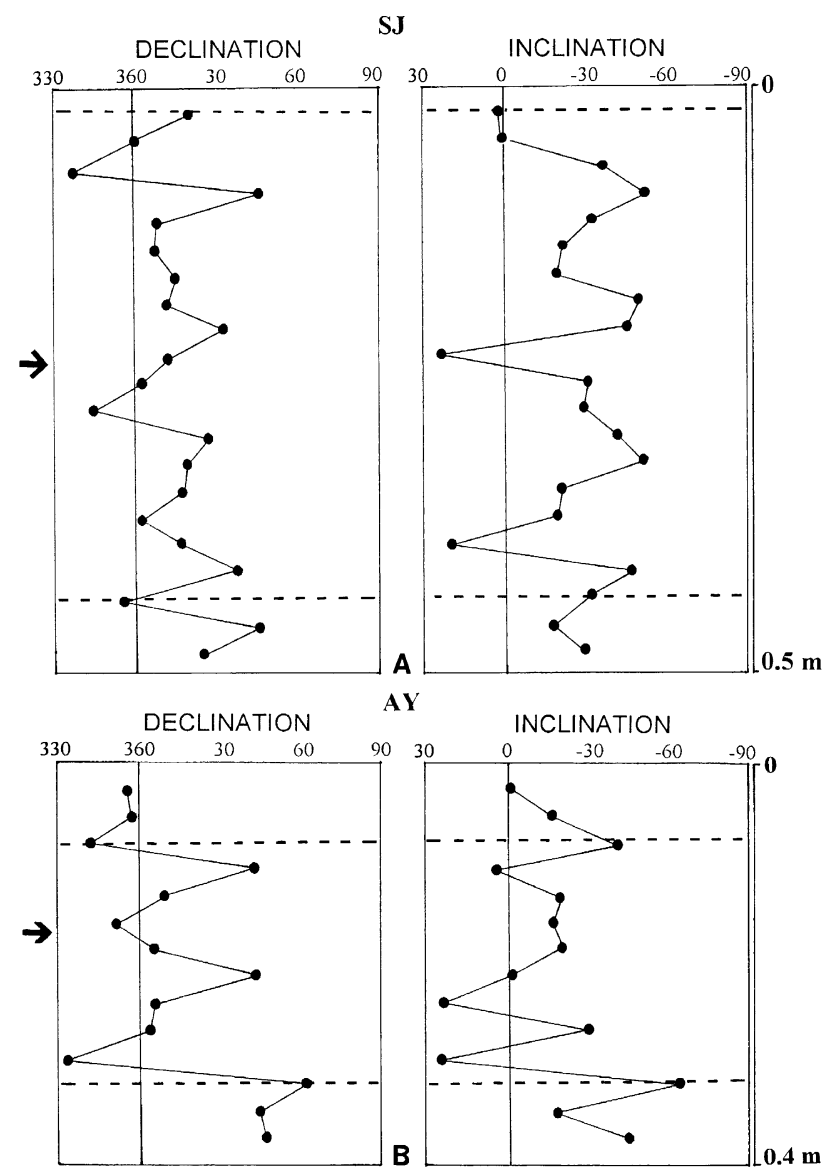

Fig. 10. Declination and inclination logs according to their stratigraphic sequence of San Juan (A) and Arroyo Yarará (B) archaeological sites. 

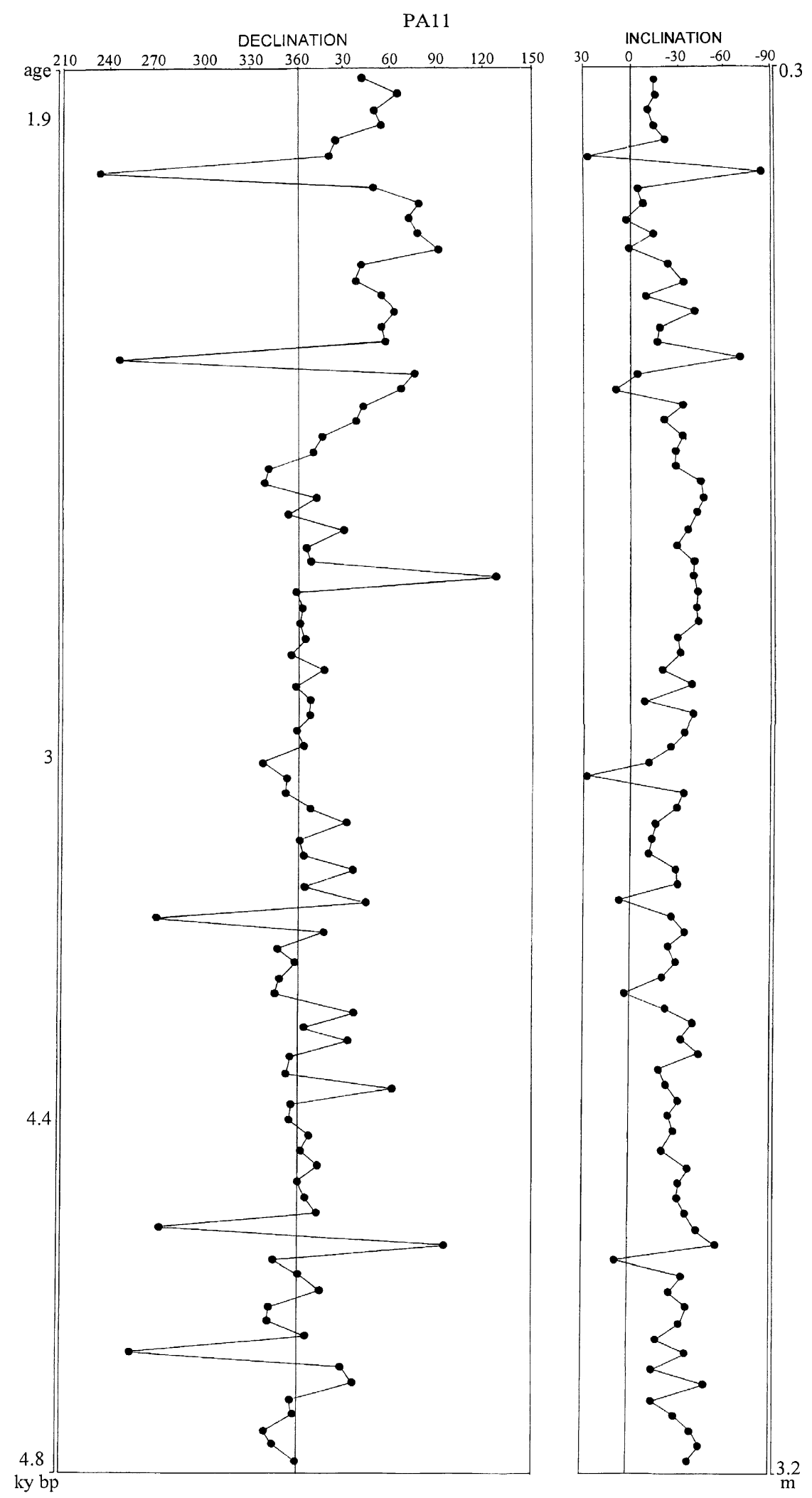

Fig. 11. Stratigraphic presentation of the profiles of declination and inclination from Piedra del Aguila 11 rockshelter related with the age of the deposit.

tions were clearly observed in Mylodon Cave and Angostura Blanca rockshelter (Fig. 18) in southern Argentina and Chile (cf. Nami, 1995). Information related to eleven records of this possible excursion are summarized in Table $3^{\dagger}$.
'Additional data might belong from the Buenos Aires province continental shelf $\left(36^{\circ} 31^{\prime} \mathrm{S}, 53^{\circ} 59^{\prime} \mathrm{W}\right)$. Nabel and Spiegelman (1988) reported reversed polarity directions that they attributed to the Matuyama Polarity Epoch. However, the sampled sediment was supposed to belong to the Holocene (Nabel, 1988). 


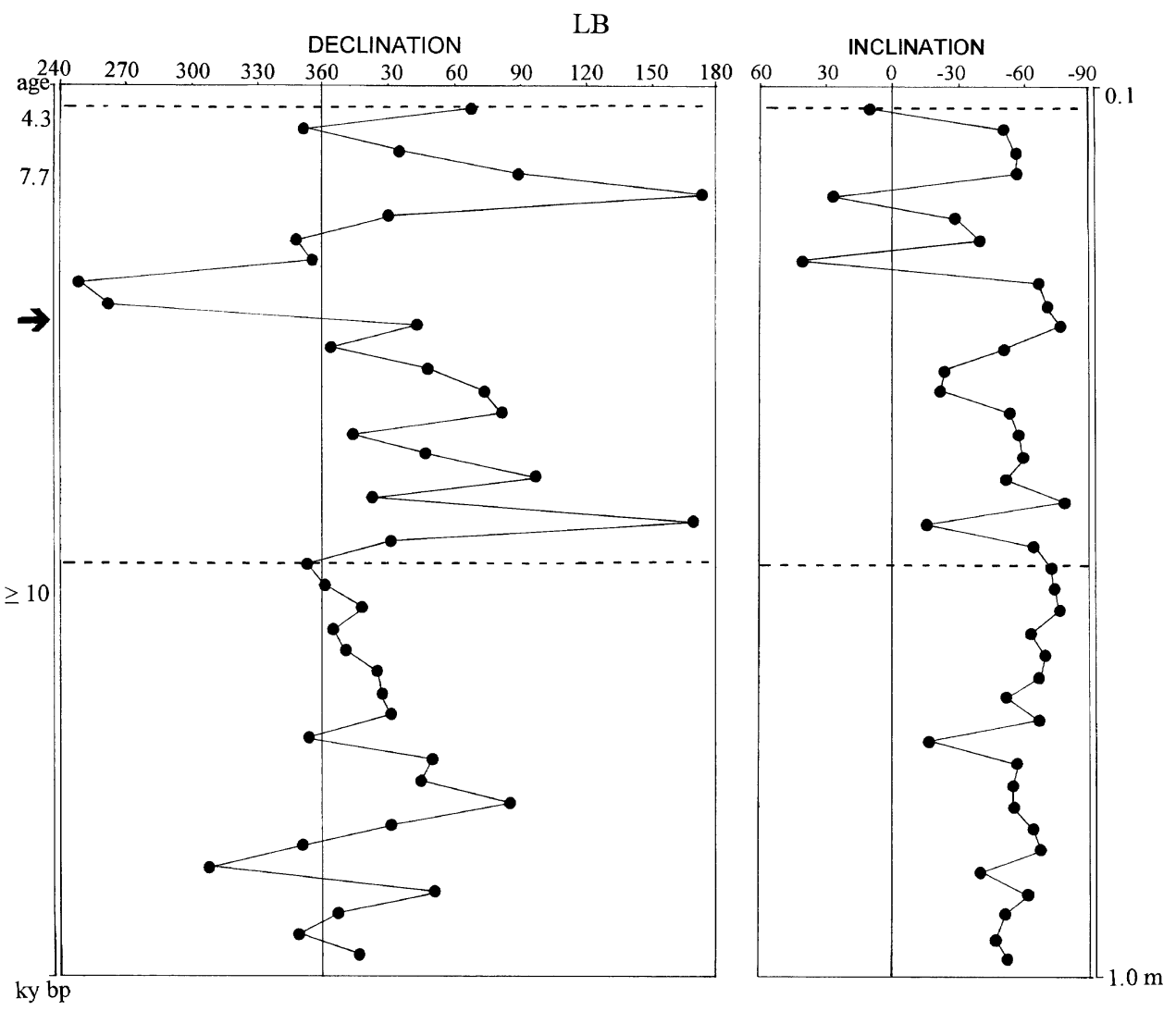

Fig. 12. Stratigraphic presentation of the declination and inclination profile from Las Buitreras Cave related with the radiocarbon dates obtained in layers IV and V.

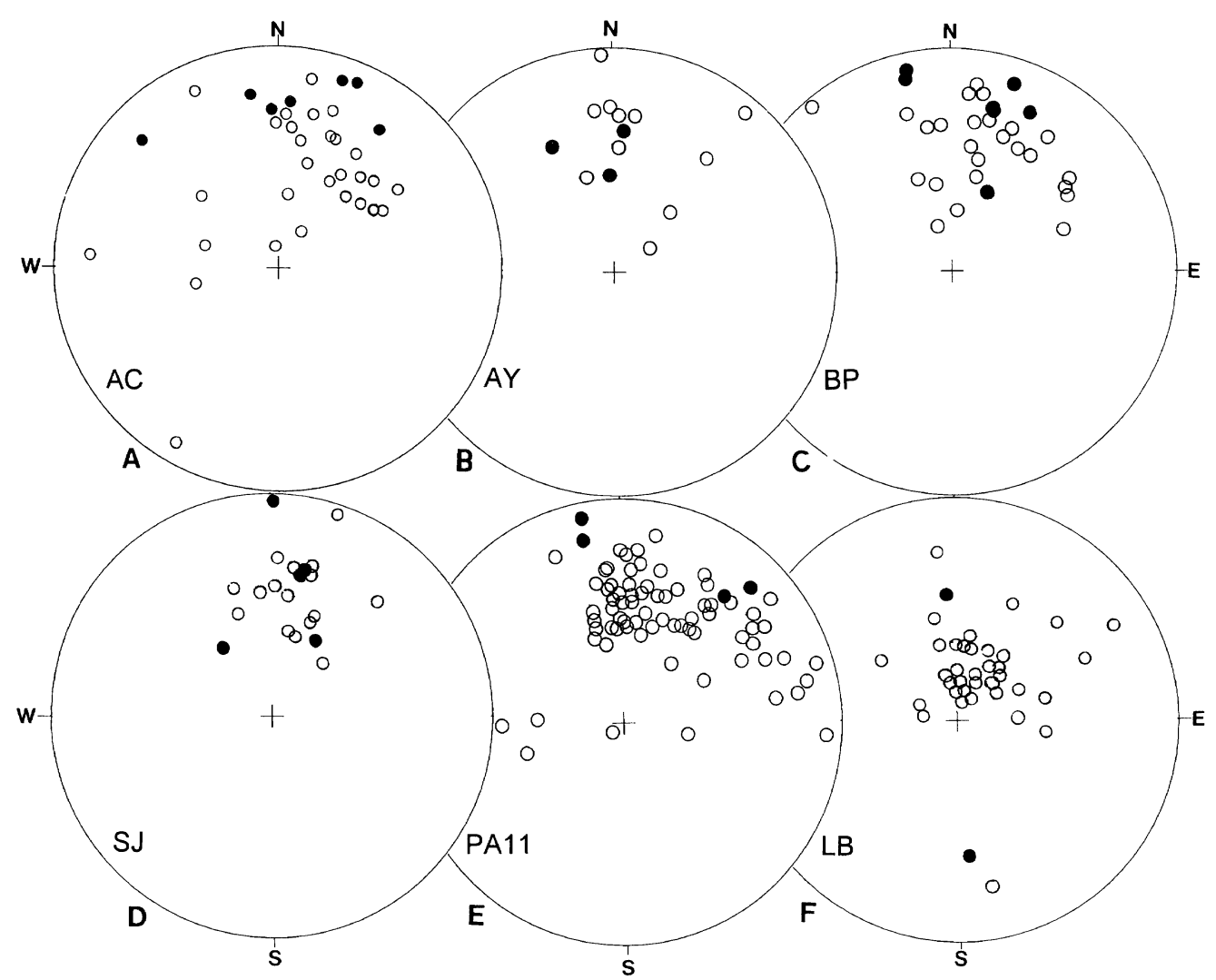

Fig. 13. Directions of characteristic remanent magnetizations (ChRM) for the sections reported in this paper. Negative inclination (open circle) and positive inclination (solid circle). 


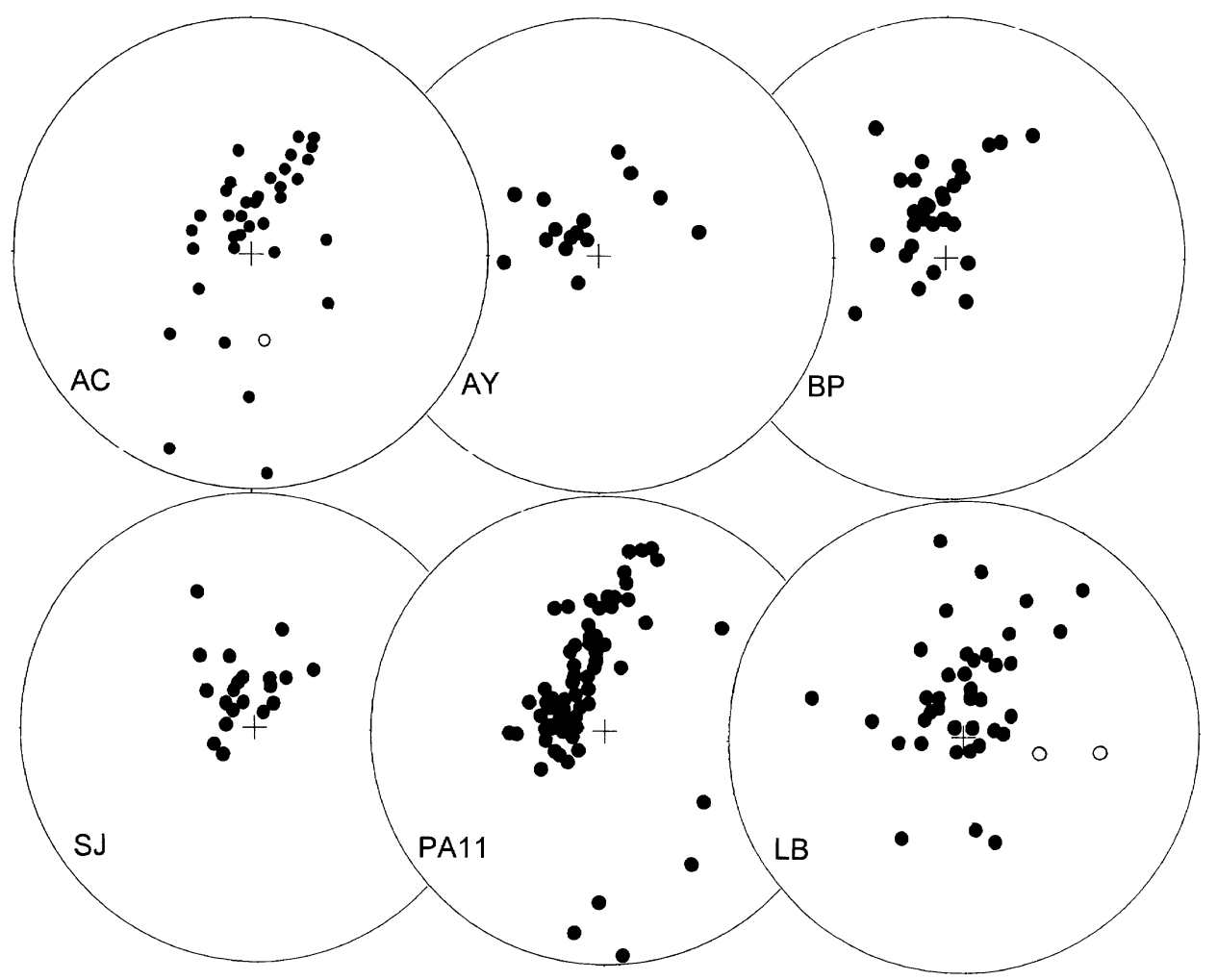

Fig. 14. Stereographic projection of virtual geomagnetic pole (VGP) calculated from directions of ChRM isolated in the sites mentioned in the text. Solid circles show those located in the northern hemisphere and open circles represented VGP located in the southern hemisphere. The centre of the projection is the geographic south pole.
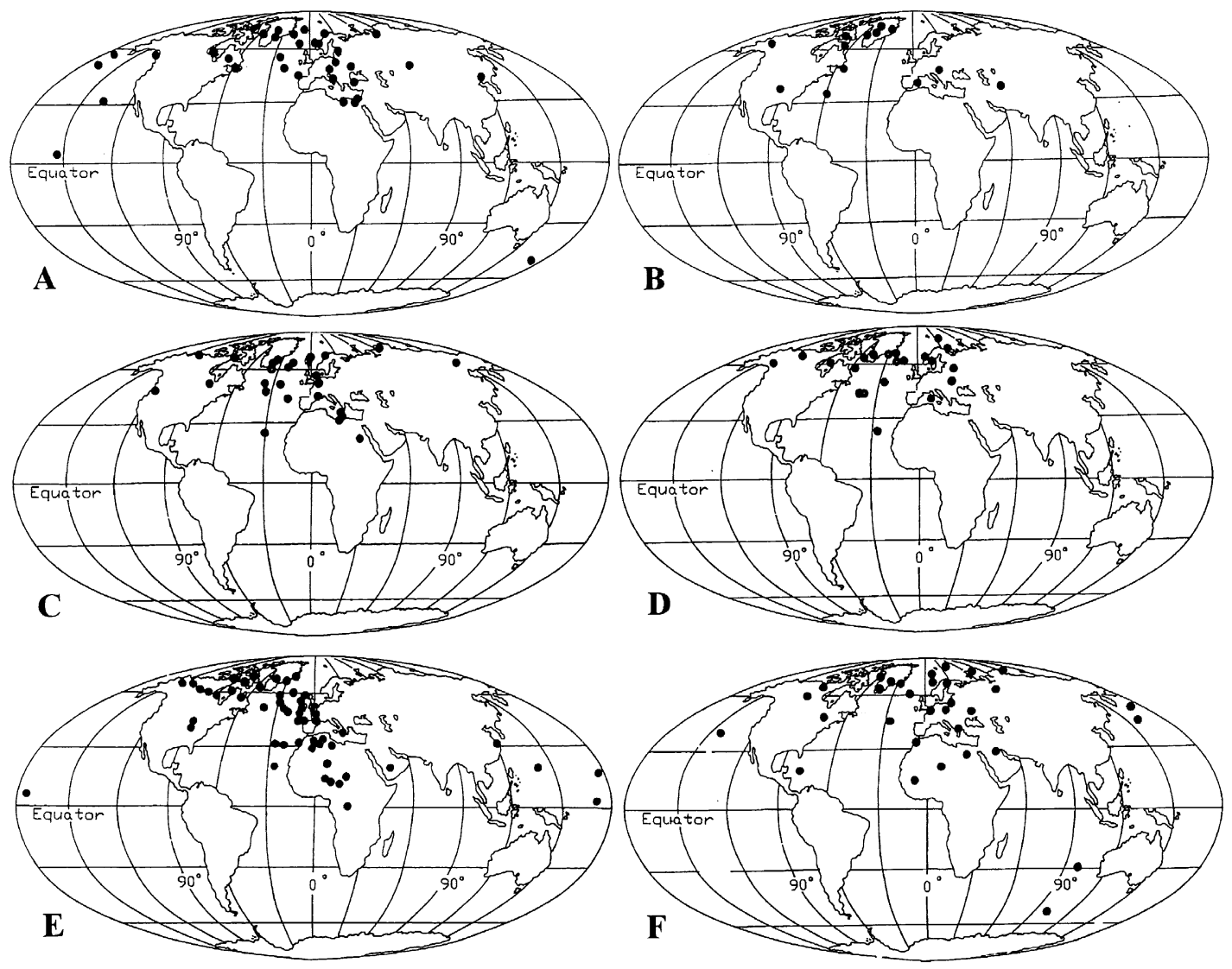

Fig. 15. VGPs paths plotted in the world map for the sites mentioned in the text. A) Alero de las Circunferencias, B) Arroyo Yarará, C) Barranca Pelada, D) San Juan, E) Piedra del Aguila 11, F) Las Buitreras. 
Table 3. Summary of archaeological sites, location, number and range of ${ }^{14} \mathrm{C}$ dates of sampled deposit, number, quality and polarity of the samples. References: $N D$ : number of ${ }^{14} \mathrm{C}$ dates in the archaeological site, $*$ : indicate indirect dates, $N$ : Number of samples used in the analysis/total number of samples treated, $\mathrm{P}$ : Polarity of the core, $\mathrm{N}=$ Normal, $\mathrm{R}=$ Reverse, $\mathrm{I}=$ Intermediate. Intermediate considered when departure from the mean is greater than $30^{\circ}$ (Quideller and Valet, 1996), SQ: Sample quality, HRe: Highly reliable, MRe: Moderately reliable, PRe: Poorly reliable.

\begin{tabular}{llccccl}
\hline \multicolumn{1}{c}{ Site } & \multicolumn{1}{c}{ Coordinates } & Age (ky bp) & $N D$ & \multicolumn{1}{c}{$\mathrm{P}$} & $N$ & \multicolumn{1}{c}{ SQ } \\
\hline Mylodon Cave & $51^{\circ} 35^{\prime} \mathrm{S} 72^{\circ} 38^{\prime} \mathrm{W}$ & $\sim 11-5.5$ & 31 & N-R & 34 & HRe \\
Cueva del Medio & $51^{\circ} 35^{\prime} \mathrm{S} 72^{\circ} 38^{\prime} \mathrm{W}$ & $\sim 11-2.1$ & 20 & N-I & 12 & MRe-PRe \\
Don Ariel & $52^{\circ} \mathrm{S} 70^{\circ} 09^{\prime} \mathrm{W}$ & $\sim 7-2.6$ & 6 & N-R & 15 & HRe-MRe \\
Angostura Blanca & $42^{\circ} 30^{\prime} \mathrm{S} 70^{\circ} \mathrm{W}$ & $\sim 2.9-2.1$ & 2 & R-N & 11 & HRe-MRe-PRe \\
A. Circunferencia & $22^{\circ} 56^{\prime} \mathrm{S} 65^{\circ} 21^{\prime} \mathrm{W}$ & $\sim 10.7-7.9$ & 5 & N-I & 36 & HRe-MRe \\
Puerto Segundo & $25^{\circ} 59^{\prime} \mathrm{S} 54^{\circ} 39^{\prime} \mathrm{W}$ & $\sim 7-0.6$ & $*$ & N-R & 17 & MRe-PRe \\
Arroyo Yarará & $26^{\circ} \mathrm{S} 55^{\circ} 21^{\prime} \mathrm{W}$ & $\sim 7-1$ & $*$ & N-I & 14 & HRe-MRe \\
Barranca Pelada & $30^{\circ} 15^{\prime} \mathrm{S} 57^{\circ} 37^{\prime} \mathrm{W}$ & $\sim 6-0.3$ & $*$ & N-I & 34 & HRe \\
San Juan & $30^{\circ} \mathrm{S} 57^{\circ} 44^{\prime} \mathrm{W}$ & $\sim 6-0.3$ & $*$ & N-I & 21 & HRe \\
Piedra del Aguila 11 & $40^{\circ} 02^{\prime} \mathrm{S} 70^{\circ} 00^{\prime} \mathrm{W}$ & $\sim 4.9-1.9$ & 9 & N-I & 90 & HRe-MRe \\
Las Buitreras & $51^{\circ} 44^{\prime} \mathrm{S} 70^{\circ} 10^{\prime} \mathrm{W}$ & $\sim 11-3$ & 4 & R-N & 40 & HRe-MRe \\
\hline
\end{tabular}

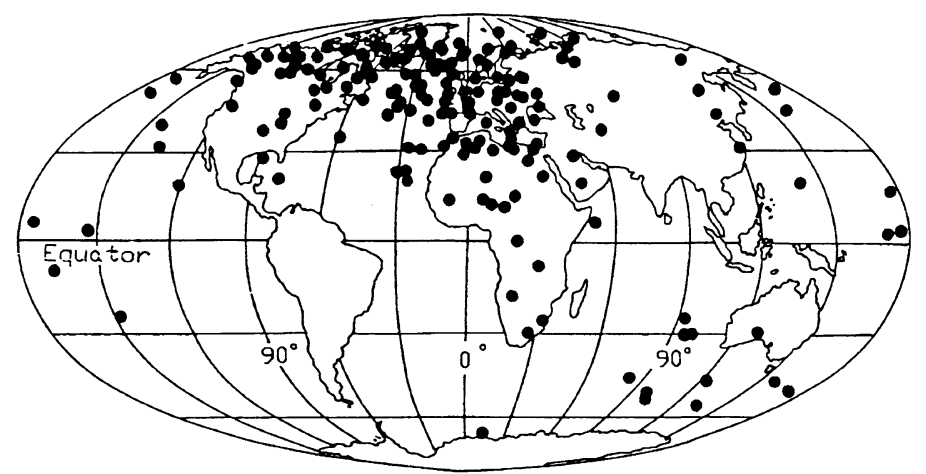

Fig. 16. World map showing the location of the VGP obtained from the sites described in Table 3

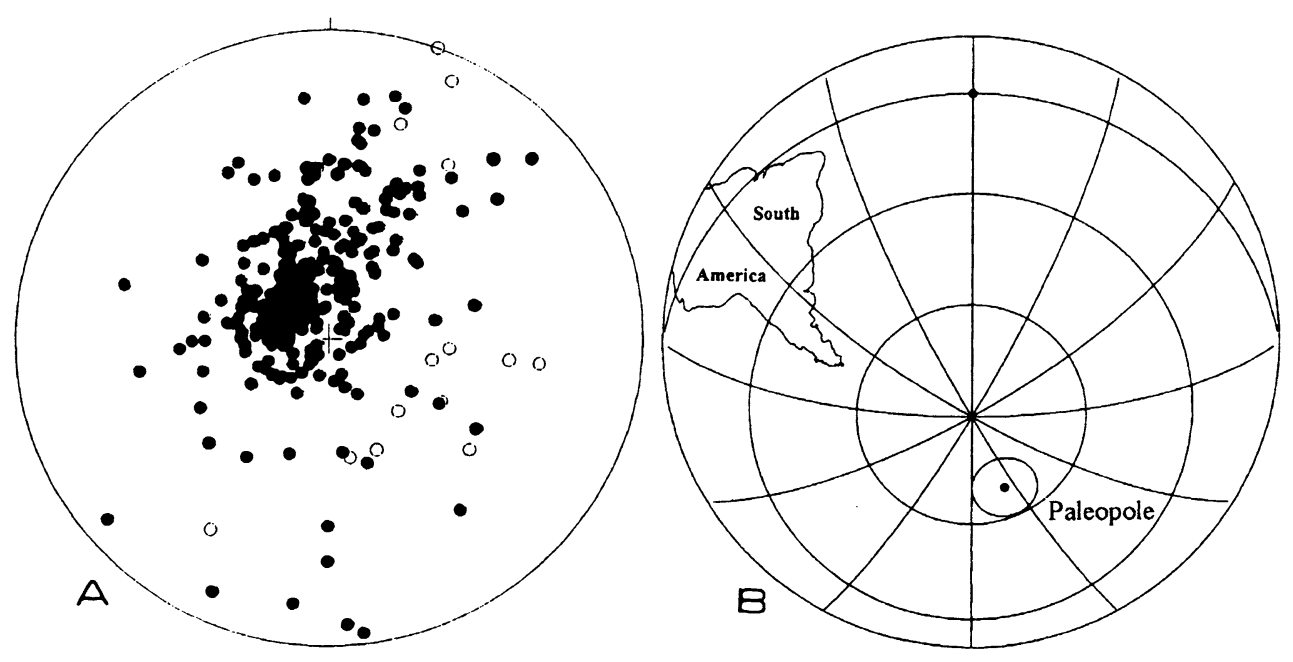

Fig. 17. A) Stereographic projection of the VGP used to isolate the palaeopole showed in B). B) Projection showing the palaeopole obtained with a $40^{\circ}$ filter and their confidence interval. 


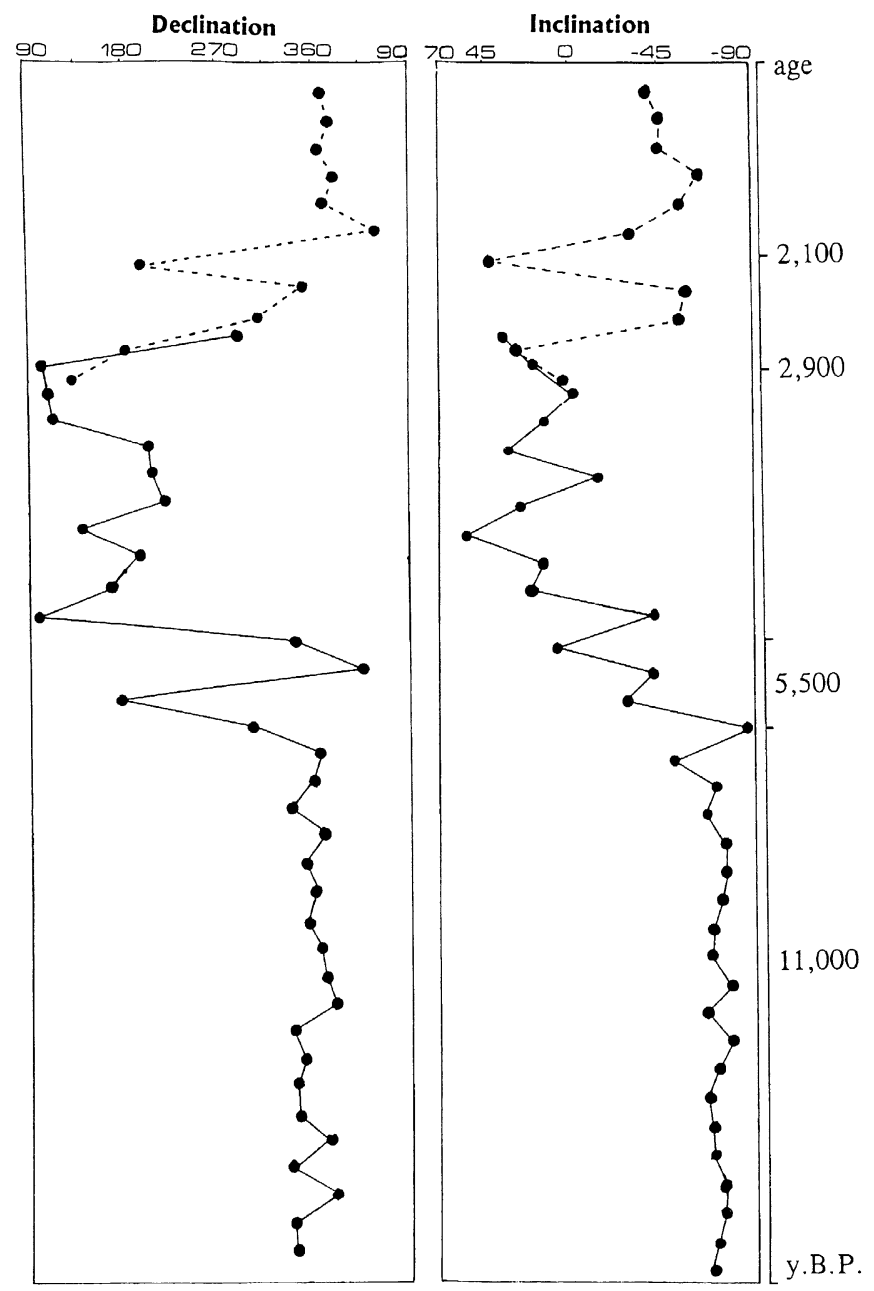

Fig. 18. Plotted declination and inclination from Angostura Blanca rockshelter (dashed line) and Mylodon Cave (continuous line) showing the N-R and $\mathrm{R}-\mathrm{N}$ geomagnetic field shift between $\leq 11$ and $\leq 2 \mathrm{ky}$ bp (from Nami, 1995).

Virtual geomagnetic pole (VGP) positions calculated only from HRe and MRe samples (Fig. 15) coincide remarkably well with transitional VGP's obtained from the sites where the GF anomalous behaviour was observed. It can be seen that they cluster near Antarctica, Australia, Africa, Europe, Eastern Asia and North America (Fig. 16).

In summary, several archaeological and palaeontological sites in southern South America have yielded records of a probable excursion of the Earth's Magnetic Field at sometime in the terminal Pleistocene and Holocene. Alternative interpretations on these records are: they represent the real evidence of the GF direction and the local fluctuations and oscillations occurred during excursions and reversions (Bogue, 1991; Bogue and Merrill, 1992) or, they result from a sediment artefact or, the influence of a delayed remanence acquisition (van Hoof and Langereis, 1991; Langereis et al., 1992, 1997). If this last alternative is true, it is possible to suppose that reversed polarity positions may have existed during that time span.

On-going analysis and putative research will provide additional information on Holocene GF behaviour in southern South America.
Acknowledgments. I am indebted to the following: National Geographic Society (Grant \# 5691-96) supported diverse aspects of the palaeomagnetic research; the National Scientific and Technological Research (CONICET) and HIDRONOR S. A. that enabled to carry out the archaeological investigations in Patagonia; Monte Caseros Municipality for their logistic support; M. I. Hernandez Llosas, J. Mujica, J. L. and A. Gherardi for they help and support during the fieldwork and information related with AC, AY, BP and SJ archaeological sites; A. C. Sanguinetti de Bórmida and D. Curzio provided very valuable information concerning PA11 and LB sites; S. Rodriguez and M. Cuadrado were very helpful during the fieldwork at LB; A. Rapalini, H. Vizán and C. Vasquez for their continuous support, help and counseling during the processing and interpretation of the paleomagnetic data. Palaeomagnetic data were processed with IAPD and MAG88 programs developed by Torsvik (Norwegian Geological Survey) and E. Oviedo (University of Buenos Aires) respectively. To one anonymous reviewer and K. M. Creer for their useful observations; and to K. M. Creer and A. Rapalini for their help and cooperation during the edition of this paper. I acknowledge support from my colleagues at the "Daniel A. Valencio" Laboratory for their fruitful discussions and invaluable help during this research.

\section{References}

Bayarski, A., A. Monti, and M. A. Gonzalez, Sedimentología del Alero Pinstoscayoc 1. Departamento Humahuaca, Jujuy, MS, 1995. Bogue, S. W., Reversals of opinion, Nature, 351, 445-446, 1991. 
Bogue, S. W. and R. T. Merrill, The character of the field during geomagnetic reversals, Annu. Rev. Earth Sci., 20, 181-219, 1992.

Borrero, L. A., La extinción de la megafauna en Patagonia, Ans. Inst. Pat. Ser. Cs. Hs., 25, 89-102, 1997.

Butler, R. F., Paleomagnetism: Magnetic Domains to Geologic Terranes, 319 pp., Blackwell Scientific Publications, Cambridge, 1992.

Creer, K. M. and J. S. Kopper, Palaeomagnetic dating of paintings in Tito Bustillo Cave, Science, 186, 348-350, 1974.

Elwood, B. B., An archeomagnetic measurement of the age and sedimentation rate of climax cave sediments, southwest Georgia, Am. J. Science, 271, 304-310, 1971.

Eugenio, E., Geoarqueología de Cueva Las Buitreras (Provincia de Santa Cruz, Argentina), Prahistoria, 3, 1997 (in press).

Hernández Llosas, M. I., H. Alem, M. P. Falchi, M. Gavilán, H. Knauth, J. B. León, P. Masci, G. Scarafia, D. Tamburini, and D. Vainstub, Proyecto arqueológico Pintoscayoc. Departamento de Humahuaca, Provincia de Jujuy, Rev. Mus. Hist. Nat. S. Raf. (Mendoza), XIV (1/4), 160-162, 1994.

Hyodo, M., C. Itota, and K. Yaskawa, Geomagnetic secular variation from magnetizations of wide-diameter cores of Holocene sediments in Japan, J. Geomag. Geoelectr., 45, 669-696, 1993.

Kirschvink, J. L., The least-squares line and plane and the analysis of palaeomagnetic data, Geophys. J. R. astr. Soc., 62, 699-718, 1980.

Kopper, J. S. and K. M. Creer, Cova dest Alexanderes, Majorca. Palaeomagnetic dating and archaeological interpretation of its sediments, Caves and Karst. Res. Speleol., 15 (2), 13-20, 1973.

Kopper, J. S. and K. M. Creer, Palaeomagnetic dating and stratigraphic interpretation in archaeology, MASCA Newsletter (Philadelphia), 12 (1), $1-4,1976$.

Langereis, C. G., A. A. M. van Hoof, and P. Rochette, Longitudinal confinement of geomagnetic reversal paths as a possible sedimentary artefact, Nature, 358, 228-230, 1992.

Langereis, C. G., M. J. Dekkers, G. J. de Lange, M. Paterne, and P. J. M. van Santvoort, Magnetostratigraphy and astronomical calibration of the last 1.1 Myr from an eastern Mediterranean piston core and dating of short events in the Brunhes, Geophys. J. Int., 129, 75-94, 1997.

Lovlie, R. and A. Sandness, Palaeomagnetic excursions recorded in midWeischselian Cave sediments from Skjonghelleren, Valderoy, W. Norway, Phys. Earth Planet. Inter., 45, 337-348, 1987.

McElhinny, M. W., B. J. J. Embleton, and P. Wellman, A synthesis of Australian cenozoic palaeomagnetic results, Geophys. J. R. astr. Soc., 36, 141-151, 1974.

Merrill, R. T. and M. W. McElhinny, The Earth's Magnetic Field. Its History, Origin and Planetary Perspective, 401 pp., Academic Press, New York, 1983.

Nabel, P., Alcances y limitaciones del paleomagnetismo en el estudio de sedimentos Holocenos: Dos ejemplos, in Resúmenes expandidos. Simposio Internacional sobre el Holoceno en América del Sur (INQUACADINQUA), edited by M. H. Iriondo and C. Ceruti, pp. 178-181, Museo de Ciencias Naturales "Profesor A. Serrano", Paraná, 1988.

Nabel, P. and A. Spiegelman, Caracterización paleomagética y sedimentológica en sedimentos de la plataforma continental externa, provincia de Buenos Aires, Actas. Seg. Jor. Geol. Bon., 307-316, 1988.

Nami, H. G., Informe sobre la segunda y tercera expedición en la Cueva del Medio. Perspectivas arqueológicas para la Patagonia Austral, Ans. Inst. Pat. Ser. Cs. Hs., 17, 71-105, 1987.

Nami, H. G., Excursiones geomagnéticas y arqueología: Nuevos datos y perspectivas en la Patagonia, Rev. Mus. Hist. Nat. S. Raf. (Mendoza), XIII (1/4), 362-367, 1994.

Nami, H. G., Holocene geomagnetic excursion at Mylodon Cave, Ultima Esperanza, Chile, J. Geomag. Geoelectr., 47, 1325-1332, 1995.

Nami, H. G., New assessments of early human occupations in the southern cone, in Prehistoric Mongoloid Dispersals, edited by T. Akazawa and E. J. Szathmáry, pp. 254-269, Oxford University Press, Oxford, 1996.

Nami, H. G. and T. Nakamura, Cronología radiocarbónica con AMS so- bre muestras de hueso procedentes del sitio Cueva del Medio (Ultima Esperanza, Chile), Ans. Inst. Pat. Ser. Cs. Hs., 23, 125-133, 1995.

Nami, H. G. and A. M. Sinito, Preliminary paleomagnetic results, the Campo Cerda Rockshelter, Province of Chubut, Argentina, Quat. South Am. Antarc. Penninsula, 9, 141-151, 1991.

Nami, H. G. and A. M. Sinito, Evidence of a possible excursion of the geomagnetic field registered during the late Holocene in the Province of Chubut, Argentina, Geoacta, 20, 19-26, 1993.

Nami, H. G., A. M. Sinito, and C. Gogorza, Primeros resultados de los estudios paleomanéticos en sedimentos de Cueva del Medio (Ultima Esperanza, Chile), Ans. Inst. Pat. Ser. Cs. Hs., 23, 135-142, 1995.

Nami, H. G., A. M. Sinito, and C. Gogorza, Observaciones paleomagnéticas en el sitio arqueológico Puerto Segundo (Misiones, Argentina), Pesquisas, Ser. Ant., 1998 (in press).

Nöel, M. and L. Thistlewood, Developments in Cave Sediments Palaeomagnetism. Geomagnetism and Palaeomagnetism, edited by F. J. Lowes, D. W. Collinson, J. H. Pary, S. K. Runcorn, D. C. Tozer, and A. Soward, pp. 91-106, Kluwer Academic Publisher, Dordrecht, 1989.

Papmarinopoulous, S. and K. Creer, The Palaeomagnetism of Cave Sediments. Geomagnetism of Backed Clays and Recent Sediments, edited by K. M. Creer, P. Tucholka, and C. E. Bartom, Elsevier, 1983.

Parés, J. M. and A. Pérez-González, Palaeomagnetic age for hominid fossils at Atapuerca archaeological site, Spain, Science, 269, 830-832, 1995.

Prieto, A., Cazadores Tempranos y Tardíos en Cueva del Lago Sofía 1, Ans. Inst. Pat. Ser. Cs. Soc., 20, 75-96, 1991.

Quideller, X. and J. P. Valet, Geomagnetic changes across the last reversal recorded in lava flows from La Palma, Canary Islands, J. Geophys. Res., 101, 13,755-13,773, 1996.

Rodriguez, J. A., Arqueología del Sudeste de Sud América, in Prehistoria de Sudamérica. Nuevas Perspectivas, edited by B. Meggers, pp. 177-209, Taraxatum, Washington, D.C., 1992.

Sanguinetti de Bórmida, A. C., Excavaciones prehistóricas en la cueva de Las Buitreras, Santa Cruz, Argentina, Rel. Soc. Arg. Ant. (Buenos Aires), X, 271-292, 1976.

Sanguinetti de Bórmida, A. C. and D. Curzio, Excavaciones arqueológicas en el sitio Piedra del Aguila 11, Prahistoria (Buenos Aires), 2, 43-100, 1996.

Saxon, E., La Prehistoria de Fuego-Patagonia: Colonización de un hábitat marginal, Ans. Inst. Pat., 7, 63-73, 1976.

Scasso, R. A., Estudio sedimentológico de la cueva Las Buitreras, Provincia de Santa Cruz, Prahistoria, 3, 1997 (in press).

Schmidt, V. A. and T. C. Partridge, An attempt to establish a magnetostratigraphic framework for sediments deposited in Sterkfontein Cave, South Africa, EOS, 72, 44, 136, 1991.

Schmitz, P. I., Prehistoric Hunter and Gatherers of Brazil, J. W. Prehistory, 1, 53-126, 1987.

Sinito, A. M., H. G. Nami, and C. Gogorza, Analysis of palaeomagnetic results from Holocene sediments sampled at archaeological excavations in South America, Quat. South Am. Antarc. Penninsula, 10, 31-44, Balkema, 1997.

Smorczewski, M., Protocolo de Análisis Físico-Químico, Laboratorio de Análisis de Suelo (INTA), MS, 1994.

Tarling, D., Palaeomagnetism, 379 pp., Chapman and Hall, New York, 1983.

Valet, J. P., C. Laj, and P. Tucholka, High-resolution sedimentary record of a geomagnetic reversal, Nature, 322, 27-32, 1986.

van Hoof, A. A. M. and C. G. Langereis, Reversal records in marine marls and delayed acquisition of remanent magnetization, Nature, 351, 223225, 1991.

Zijderveld, J. D. A., AC demagnetization of rocks: Analysis of results, in Methods in Paleomagnetism, edited by D. W. Collinson, K. M. Creer, and S. K. Runcorn, pp. 254-286, Elsevier, 1967.

H. G. Nami (e-mail: nami@gl.fcen.uba.ar) 